\title{
The effect of solar zenith angle on MODIS cloud optical and microphysical retrievals within marine liquid water clouds
}

\author{
D. P. Grosvenor ${ }^{1, *}$ and R. Wood ${ }^{1}$ \\ ${ }^{1}$ Department of Atmospheric Science, University of Washington, Seattle, USA \\ *now at: School of Earth and Environment, University of Leeds, UK \\ Correspondence to: D. P. Grosvenor (daniel.p.grosvenor@gmail.com)
}

Received: 13 September 2013 - Published in Atmos. Chem. Phys. Discuss.: 7 January 2014

Revised: 14 May 2014 - Accepted: 5 June 2014 - Published: 17 July 2014

\begin{abstract}
In this paper we use a novel observational approach to investigate MODIS satellite retrieval biases of $\tau$ and $r_{\mathrm{e}}$ (using three different MODIS bands: 1.6, 2.1 and $3.7 \mu \mathrm{m}$, denoted as $r_{\mathrm{e} 1.6}, r_{\mathrm{e} 2.1}$ and $r_{\mathrm{e} 3.7}$, respectively) that occur at high solar zenith angles $\left(\theta_{0}\right)$ and how they affect retrievals of cloud droplet concentration $\left(N_{\mathrm{d}}\right)$. Utilizing the large number of overpasses for polar regions and the diurnal variation of $\theta_{0}$ we estimate biases in the above quantities for an open ocean region that is dominated by low level stratiform clouds.

We find that the mean $\tau$ is fairly constant between $\theta_{0}=$ $50^{\circ}$ and $\sim 65-70^{\circ}$, but then increases rapidly with an increase of over $70 \%$ between the lowest and highest $\theta_{0}$. The $r_{\mathrm{e} 2.1}$ and $r_{\mathrm{e} 3.7}$ decrease with $\theta_{0}$, with effects also starting at around $\theta_{0}=65-70^{\circ}$. At low $\theta_{0}$, the $r_{\mathrm{e}}$ values from the three different MODIS bands agree to within around $0.2 \mu \mathrm{m}$, whereas at high $\theta_{0}$ the spread is closer to $1 \mu \mathrm{m}$. The percentage changes of $r_{\mathrm{e}}$ with $\theta_{0}$ are considerably lower than those for $\tau$, being around $5 \%$ and $7 \%$ for $r_{\mathrm{e} 2.1}$ and $r_{\mathrm{e} 3.7}$. For $r_{\mathrm{e} 1.6}$ there was very little change with $\theta_{0}$. Evidence is provided that these changes are unlikely to be due to any physical diurnal cycle.

The increase in $\tau$ and decrease in $r_{\mathrm{e}}$ both contribute to an overall increase in $N_{\mathrm{d}}$ of $40-70 \%$ between low and high $\theta_{0}$. Whilst the overall $r_{\mathrm{e}}$ changes are quite small, they are not insignificant for the calculation of $N_{\mathrm{d}}$; we find that the contributions to $N_{\mathrm{d}}$ biases from the $\tau$ and $r_{\mathrm{e}}$ biases were roughly comparable for $r_{\mathrm{e} 3.7}$, although for the other $r_{\mathrm{e}}$ bands the $\tau$ changes were considerably more important. Also, when considering only the clouds with the more heterogeneous tops, the importance of the $r_{\mathrm{e}}$ biases was considerably enhanced for both $r_{\mathrm{e} 2.1}$ and $r_{\mathrm{e} 3.7}$.
\end{abstract}

When using the variability of $1 \mathrm{~km}$ resolution $\tau$ data $\left(\gamma_{\tau}\right)$ as a heterogeneity parameter we obtained the expected result of increasing differences in $\tau$ between high and low $\theta_{0}$ as heterogeneity increased, which was not the case when using the variability of $5 \mathrm{~km}$ resolution cloud top temperature $\left(\sigma_{\text {СТT }}\right)$, suggesting that $\gamma_{\tau}$ is a better predictor of $\tau$ biases at high $\theta_{0}$ than $\sigma_{\text {CTT }}$. For a given $\theta_{0}$, large decreases in $r_{\mathrm{e}}$ were observed as the cloud top heterogeneity changed from low to high values, although it is possible that physical changes to the clouds associated with cloud heterogeneity variation may account for some of this. However, for a given cloud top heterogeneity we find that the value of $\theta_{0}$ affects the sign and magnitude of the relative differences between $r_{\mathrm{e} 1.6}, r_{\mathrm{e} 2.1}$ and $r_{\mathrm{e} 3.7}$, which has implications for attempts to retrieve vertical cloud information using the different MODIS bands. The relatively larger decrease in $r_{\mathrm{e} 3.7}$ and the lack of change of $r_{\mathrm{e} 1.6}$ with both $\theta_{0}$ and cloud top heterogeneity suggest that $r_{\mathrm{e} 3.7}$ is more prone to retrieval biases due to high $\theta_{0}$ than the other bands. We discuss some possible reasons for this.

Our results have important implications for individual MODIS swaths at high $\theta_{0}$, which may be used for case studies for example. $\theta_{0}$ values $>65^{\circ}$ can occur at latitudes as low as $28^{\circ}$ in mid-winter and for higher latitudes the problem will be more acute. Also, Level-3 daily averaged MODIS cloud property data consist of the averages of several overpasses for the high latitudes, which will occur at a range of $\theta_{0}$ values. Thus, some biased data are likely to be included. It is also likely that some of the $\theta_{0}$ effects described here would apply to $\tau$ and $r_{\mathrm{e}}$ retrievals from satellite instruments that use visible light at similar wavelengths along with forward retrieval models that assume plane parallel clouds, such as the GOES imagers, SEVIRI, etc. 


\section{Introduction}

The MODIS (Moderate Resolution Imaging Spectroradiometer) instruments onboard the Aqua and Terra polar orbiting satellites are capable of retrieving cloud optical depth $(\tau)$ and cloud top effective radius $\left(r_{\mathrm{e}}\right)$ information from liquid clouds based upon the combination of one non-absorbing optical wavelength $(0.86 \mu \mathrm{m}$ is used by MODIS for retrievals over the ocean) and one absorbing near-infrared band (Foot, 1988; Nakajima and King, 1990; King et al., 1997; Platnick et al., 2003); this can be either $1.6,2.1$ or $3.7 \mu \mathrm{m}$. The $r_{\mathrm{e}}$ retrieved using these different bands will hereafter be referred to, respectively, as $r_{\mathrm{e} 1.6}, r_{\mathrm{e} 2.1}$ and $r_{\mathrm{e} 3.7}$, with $r_{\mathrm{e} 2.1}$ being the value provided as standard from MODIS (e.g. in Level3 products). This information is invaluable for a range of cloud microphysical studies, especially given the global coverage and the long time period of the data set available from these instruments (Terra MODIS since mid-2000 and Aqua MODIS since 2002).

Additionally, this information can be used to estimate cloud droplet number concentrations $\left(N_{\mathrm{d}}\right)$ within liquid clouds (Boers et al., 2006; Bennartz, 2007, hereafter B07). $N_{\mathrm{d}}$ is a very useful parameter since in non-precipitating clouds it depends mainly upon the concentration of available cloud condensation nuclei (CCN), although to a lesser extent it also depends upon the cloud updraft speed. Thus, for nonprecipitating clouds with fixed updraft speeds, $N_{\mathrm{d}}$ is a good indicator of available CCN concentrations. Parameters like $r_{\mathrm{e}}$ alone are not as useful in this regard since $r_{\mathrm{e}}$ is dependent on both $N_{\mathrm{d}}$ and the local liquid water content of the cloud, which may both be variable. This makes an $N_{\mathrm{d}}$ data set useful for estimates of aerosol indirect effects (AIEs) - see for example Nakajima et al. (2001) and Quaas et al. (2008). Precipitation can also be an important sink process for $N_{\mathrm{d}}$ and, therefore, insight into such processes can be gained through knowledge of $N_{\mathrm{d}}$ (e.g. Wood et al., 2012).

A global long-term data set of $N_{\mathrm{d}}$ would also allow the evaluation of the representation of AIEs in global models, something that cannot be reliably achieved from ground and aircraft measurements with their generally poor spatial and temporal sampling. The representation of AIEs in climate models is complex and involves interactions between several processes. Thus, simulating it is a strong test for climate models. However, there are large variations of AIE estimates between different climate models (Quaas et al., 2009; IPCC, 2007) demonstrating large uncertainties in the understanding of these processes and therefore large uncertainties in the predicted climate forcing.

Marked differences in predicted $N_{\mathrm{d}}$ also exist between different GCMs, which is a good indicator that models are not correctly capturing the key controls on $N_{\mathrm{d}}$. This is likely to result in poor prediction of AIEs. Using observations of $N_{\mathrm{d}}$ to evaluate and constrain $N_{\mathrm{d}}$ in models might give insight into how to improve this situation. Additionally, many climate models arbitrarily fix a lower limit for $N_{\mathrm{d}}$ (Hoose et al.,
2009; Quaas et al., 2009). This has been shown to affect the strength of predicted AIEs across GCMs; in one model removing this limit changed the global AIE by $80 \%$ (Hoose et al., 2009). Satellite-based measurements of $N_{\mathrm{d}}$ might represent a way to determine this lower limit (if one exists).

However, there are problems with satellite retrievals and they need to be assessed before a robust and reliable $N_{\mathrm{d}}$ data set can be produced. This paper aims to examine some aspects of these problems, in particular issues that occur when retrievals are made at high solar zenith angle (SZA, or $\left.\theta_{0}\right)$. There have been a number of studies that have examined optical depth artifacts for non-absorbing wavelength retrievals at high $\theta_{0}$, which will be discussed in Sect. 2.1. However, previous observational studies have generally relied upon seasonal and/or latitudinal variations in $\theta_{0}$ to assess changes in retrieved cloud properties and it is not clear whether physical cloud properties are invariant seasonally and at different latitudes. Also, the effect on MODIS retrievals has not been studied, likely due to the difficulty in obtaining an objective test. Here we attempt such a study and extend the analysis to examine issues with $r_{\mathrm{e}}$ and $N_{\mathrm{d}}$ retrievals.

The paper is organized as follows: Sect. 2 contains a discussion on what is known from the previous literature about the effects of cloud heterogeneity and $\theta_{0}$ on cloud retrievals; Sect. 3 describes the methods, which includes a description of the method used to estimate $N_{\mathrm{d}}$ and a discussion the validity of some of the assumptions required (Sect. 3.1 and Appendix A); the method that we use here to estimate the effects of $\theta_{0}$ on $\tau, r_{\mathrm{e}}$ and $N_{\mathrm{d}}$ retrievals is described in Sect. 3.3; Sect. 4 describes the main results, including the effect of $\theta_{0}$ and also the effect of cloud top temperature heterogeneity; Sect. 5 discusses potential causes of the effects observed; and Sect. 6 provides a summary and discusses some of the ramifications of the results for the MODIS data set.

\section{Potential optical retrieval artifacts}

The $N_{\mathrm{d}}$ retrieval method (see Sect. 3.1 and Appendix A) is based upon measurements of $\tau$ and $r_{\mathrm{e}}$. We now discuss potential artifacts for $\tau$ and $r_{\mathrm{e}}$ retrievals in some detail since these are important in attempting to understand the high $\theta_{0}$ biases investigated here.

A large cause of potential artifacts is likely due to the use of the plane parallel (PP) radiative transfer algorithm that is used to build look-up tables (LUTs) for converting pairs of non-absorbing wavelength reflectance $\left(R_{\mathrm{nab}}\right)$ and absorbing wavelength reflectance $\left(R_{\mathrm{ab}}\right)$ into $\tau$ and $r_{\mathrm{e}}$ values (Nakajima and King, 1990). This requires that the clouds are horizontally homogeneous both within a single $1 \mathrm{~km} \times 1 \mathrm{~km}$ MODIS pixel and at scales outside of a given pixel. The latter is required because the PP approximation requires that each pixel be unaffected by any other pixel (the independent column approximation, ICA). Under conditions where 3-D radiative transfer of light occurs in a non-homogeneous environment, 
net horizontal photon transport can occur and thus this assumption breaks down. The assumption of no variability at scales below that of a MODIS pixel $(1 \mathrm{~km} \times 1 \mathrm{~km})$ has also been shown to be untrue for real clouds (e.g. Zhang et al., 2012, hereafter Z12). In fact, Liquid Water Content (LWC) variability of clouds has been shown to extend down to scales smaller than $4 \mathrm{~cm}$ (Marshak et al., 1998), although that study suggested that variability below the scale of the mean free path of photons in clouds ( $\sim 10-30 \mathrm{~m}$ for stratocumulus) was not important for remote-sensing applications.

The breakdown of either of these assumptions can lead to biases in the retrieved optical properties, although assessment of the direction and magnitude of these effects is complicated. MODIS optical property retrievals are made using reflectances and the effect of cloud heterogeneity on these depends on the solar and viewing geometry; i.e. upon $\theta_{0}$, the viewing zenith angle (VZA) and on the relative azimuth angle $(\phi)$. Much more work on the effects on $\tau$ than on $r_{\mathrm{e}}$ has been reported. However, any artifacts of the $r_{\mathrm{e}}$ retrieval are likely to be important for $N_{\mathrm{d}}$ calculations because of the strong sensitivity of $N_{\mathrm{d}}$ to $r_{\mathrm{e}}$ that is inherent in Eq. A1 (see Sect. 3.1).

\subsection{Optical depth retrieval artifacts}

Cahalan et al. (1994) showed that the non-linearity of the relationship between $R_{\text {nab }}$ and $\tau$ causes a decrease in albedo for heterogeneous clouds compared to a PP cloud with the same mean $\tau$. This is known as the plane parallel (PP) albedo bias and is likely to lead to $\tau$ underestimates made using the measured reflectances and PP LUTs. Also, at near-nadir viewing angles and for low $\theta_{0}$, cloud variability is known to cause the mean reflectance of a region to be slightly reduced compared to a homogeneous cloud with the same mean $\tau$ via 3-D effects, due to the leakage of photons horizontally from the sides of the region and due to channelling of photons from regions of high extinction to regions of low extinction where they can be lost through downward transport (Loeb et al., 1997; Davies, 1978; Kobayashi, 1993; Varnai and Davies, 1999). However, these biases are generally small compared to those that have been reported at high $\theta_{0}$.

Studies using data from the ERBE (Loeb and Davies, 1996, 1997) and AVHRR (Loeb and Coakley, 1998) satellites have demonstrated that at high $\theta_{0}\left(\theta_{0} \gtrsim 65^{\circ}\right)$ the optical depth inferred from the observations increased with $\theta_{0}$. This was attributed to the increasing (positive) difference in reflectances between the real observed clouds and those calculated from the PP model as $\theta_{0}$ increased. The results were found to be very sensitive to the thickness of the cloud with higher biases reported for the more optically thick clouds; for $\tau>12$ and nadir viewing the positive bias was present even at low $\theta_{0}$.

Modelling studies of $\theta_{0}$ biases are less prone to the problems inherent in satellite studies caused by assumptions about the cloud population at low and high $\theta_{0}$ being simi- lar, since the modelled cloud field is known. Using Monte Carlo 3-D radiative transfer modelling Loeb et al. (1997) showed that 3-D nadir reflectances increase with $\theta_{0}$, whereas reflectances calculated using the PP approximation decrease. This was consistent with the above observational studies indicating that 3-D radiative transfer effects within a heterogeneous cloud environment were the cause. Sensitivity tests suggested a roughly equal contribution to the bias from cloud side illumination effects and cloud top height variability effects, with the latter effect attributed to changes in the slope of cloud elements at cloud top. Such effects occurred even for completely overcast scenes. It was also indicated that cloud top height variability was more important than extinction variability. Similar conclusions were found from the modelling results of Varnai and Davies (1999).

One limitation of these modelling studies is that only nadir views were tested. By examining differences between nadir and off-nadir MISR retrievals, Liang and Girolamo (2013) found that $\tau$ retrievals are likely to be affected by VZA and $\phi$, although the effects were observed to be complicated and the sign and magnitude of the biases was suggested to be dependent upon many competing factors. However, significant $\tau$ biases were generally not seen until very high VZA values of $70.5^{\circ}$ were reached; biases within the MODIS VZA range were much lower. It was also found that cloud heterogeneity tended to enhance the magnitude of the effects, particularly for low optical depth clouds and at high $\theta_{0}$.

Finally, Seethala and Horvath (2010) found that MODISderived Liquid Water Path (LWP) measurements increased significantly relative to co-located measurements from AMSR-E (Advanced Microwave Scanning RadiometerEOS) at high $\theta_{0}$. A large part of this was attributed to unphysical increases in $\tau$ with $\theta_{0}$. The increase was greater as the inhomogeneity of MODIS $\tau$ over the $0.25^{\circ} \times 0.25^{\circ}$ scenes increased, which is consistent with the above results.

\subsection{Effective radius retrieval artifacts}

Whilst there have been a number of studies examining the effects of cloud variability and viewing geometry on $\tau$ retrievals there have been far fewer studies on the $r_{\mathrm{e}}$ effect. Marshak et al. (2006, hereafter M06) was one of the first to do so and introduced a theoretical basis to attempt to explain the effects of 3-D radiative transfer on $r_{\mathrm{e}}$ retrievals that were made using cloud fields from an LES (Large Eddy Simulation) model. M06 divided the effects into those due to resolved variability of reflectances (i.e. variability at scales larger than the satellite pixel size) and those due to subpixelscale variability.

For resolved scale variability the theory suggested that 3$D$ radiative transfer effects were expected to lead to a tendency for an overall increase in $r_{\mathrm{e}}$ and $\tau$ (relative to the true values) due to the non-linearity of the relationship between the reflectances and $r_{\mathrm{e}}$ and $\tau$. M06 suggested that subpixel variability would lead to a low bias of both the $\tau$ and $r_{\mathrm{e}}$ 
values retrieved for that pixel due to averaging of the reflectances prior to the retrieval of $\tau$ and $r_{\mathrm{e}}$ (a satellite viewing the pixel would report the averaged reflectance). The theory stipulated a number of assumptions that are unlikely to hold true in all circumstances. Nevertheless, the results from the retrievals made from reflectances calculated from the LES cloud model fields corroborated the theoretical arguments, suggesting that, at least in this case, the assumptions may have been valid, or irrelevant.

However, also using retrievals performed on LES clouds, Z12 found the opposite result for the effect of subpixel averaging of reflectances, with the $r_{\mathrm{e}}$ retrieved at $800 \mathrm{~m} \times 800 \mathrm{~m}$ resolution (close to the size of a $1 \mathrm{~km} \times 1 \mathrm{~km}$ MODIS pixel) being larger than the mean calculated from the $100 \mathrm{~m} \times 100 \mathrm{~m}$ grid cells of the model. It was found that the $r_{\mathrm{e}}$ within the $800 \mathrm{~m} \times 800 \mathrm{~m}$ regions was approximately constant, but that there was quite a wide spread in $\tau$. This was also demonstrated for a limited sample of real clouds using MODIS observations. Z12 showed that for such variability the 2-D nature of the dual-band LUT used for MODIS retrievals would lead to increases in $r_{\mathrm{e}}$ (and decreases in $\tau$ ) and that the increase would be greater as the subpixel heterogeneity of $R_{\text {nab }}$ increased. For the cases considered, these results negated the assumption of independence of the $\tau$ and $r_{\mathrm{e}}$ retrievals made in M06 since the subpixel $\tau$ variability meant that the nonorthogonal regions of the LUT were utilized. Thus it remains to be explained why the results from the LES model simulations in M06 were consistent with that theoretical basis.

One major difference between the simulations of M06 and Z12 that might provide a potential explanation is that the radiative transfer on the cloud fields from the M06 simulations were performed at the moderately high $\theta_{0}$ of $60^{\circ}$, whereas in Z12 radiative transfer was performed at $\theta_{0}=20$ and $50^{\circ}$ and on the whole results were reported for the combination of the two $\theta_{0}$ values. It is likely that the result obtained will depend on the degree of subpixel variation of both $R_{\mathrm{nab}}$ and $R_{\mathrm{ab}}$, the region of the LUT covered by the reflectance values and the influence on the subpixel reflectances of 3-D effects. Such factors are likely to be affected by the value of $\theta_{0}$. Other factors that alter the orthogonality and non-linearity of the LUTs are also likely to affect this result, such as the nearinfrared wavelength used, as also demonstrated in Z12. Their results showed that the increase of $r_{\mathrm{e}}$ due to subpixel averaging was substantially greater for the $2.1 \mu \mathrm{m}$ band relative to the $3.7 \mu \mathrm{m}$ band, and that this was most likely because the LUT for the latter is more orthogonal than for the former.

There have been several attempts in the literature to use the differences between $r_{\mathrm{e}}$ from the different MODIS bands to infer information about the vertical structure of the cloud. This may be theoretically possible since the different wavelengths of light have different penetration depths into the cloud and thus produce a weighted mean $r_{\mathrm{e}}$ that is representative of different vertical regions of the cloud (Platnick, 2000). However, the heterogeneity effects just mentioned will clearly impact such attempts. Further discussion on this is deferred to Sects. 4.2.2 and 5.3.

\section{Methods}

\subsection{The method used to estimate droplet concentration}

The method used for the estimation of $N_{\mathrm{d}}$ from MODIS $\tau$ and $r_{\mathrm{e}}$ measurements follows that described in Boers et al. (2006) and B07. Details about this, including necessary assumptions and their justification, are discussed in Appendix A.

\subsection{Measures of cloud heterogeneity}

Given the sensitivity of the cloud optical retrievals to cloud inhomogeneity it is desirable to restrict them to regions that are as homogeneous as possible. It seems that restricting analysis to regions where the MODIS cloud fraction is high is one way to increase the probability of homogeneity, since it was shown by Wood and Hartmann (2006) that, over the scale of $\sim 200 \mathrm{~km}$, cloud fraction is strongly correlated with a measure of homogeneity based on the MODIS liquid water path (denoted $\gamma_{\text {LWP }}$ ). However, the degree of variability at scales smaller than the MODIS $1 \mathrm{~km} \times 1 \mathrm{~km}$ pixel size was not assessed. Additionally, it has been shown that inhomogeneities within completely overcast stratocumulus may still introduce retrieval artifacts (Loeb et al., 1997).

Painemal and Zuidema (2011, hereafter PZ11) restricted their validation of MODIS microphysical retrievals (see Appendix A for further details on this) to regions that had cloud fractions $>90 \%$ over a $5 \mathrm{~km} \times 5 \mathrm{~km}$ region (note, the $5 \mathrm{~km} \times 5 \mathrm{~km}$ cloud mask is a standard MODIS product). Using another metric, the subpixel heterogeneity index, defined in Zhang and Plantnick (2011) as the ratio between the spatial standard deviation and mean of the $0.86 \mu \mathrm{m}$ reflectance over an area of $1 \mathrm{~km} \times 1 \mathrm{~km}$, PZ11 found that such $>90 \%$ cloud fraction regions were generally very homogeneous by this measure. However, this quantity only measures the subpixelscale variability of the clouds. Variability over larger scales was not examined in PZ11 and open questions remain concerning the scale over which homogeneity is required in order to avoid 3-D radiative biases (within acceptable tolerances).

In line with other studies, PZ11 found that, on average, MODIS $r_{\mathrm{e}}$ values were 15-20\% too large compared to the in situ observations, despite the reported subpixel homogeneity. The reason for this discrepancy was not established, although it can be speculated that a combination of the types of effects discussed above (3-D radiative transfer and subpixel averaging of reflectances) may be to blame. The results also suggest that ensuring low subpixel $R_{\text {nab }}$ heterogeneity does not mean that $r_{\mathrm{e}}$ biases will be avoided. 
Following Cahalan et al. (1994), Seethala and Horvath (2010) assessed cloud homogeneity from MODIS scenes over larger scales $\left(0.25^{\circ} \times 0.25^{\circ}\right)$ using a measure of $\tau$ variability:

$\gamma_{\tau}=1-\frac{\exp (\overline{\ln \tau})}{\bar{\tau}}$

where $\gamma_{\tau}$ varies from 0 to 1 , with larger values denoting more heterogeneity. We also use this measure of heterogeneity in the current work, except that we calculate it over $1^{\circ} \times 1^{\circ}$ regions. However, a difficulty with the measures of cloud heterogeneity mentioned so far is that they depend on reflectance variability. Variability in reflectance has been shown to be caused by viewing geometry variations (particularly due to high $\theta_{0}$ ) and so this is not always a measure of actual physical cloud variability; it is useful to be able to separate these two effects.

In this paper we mainly use the standard deviation of the MODIS cloud top temperature (CTT) over a $1^{\circ} \times 1^{\circ}$ region, $\sigma_{\mathrm{CTT}}$, to characterize heterogeneity. This will not be affected by optical artifacts, as would be the case for $\gamma_{\tau}$ and $\gamma_{\mathrm{LWP}}$ and thus should be more representative of the physical cloud heterogeneity. This measure also has the advantage that it will characterize cloud top heterogeneities, whereas the other measures could also be affected by e.g. extinction variability within cloud; the studies mentioned in Sect. 2.1 (Loeb et al., 1997; Varnai and Davies, 1999) found that cloud top height variability had a larger effect on cloud reflectance than did extinction variability. However, $\sigma_{\mathrm{CTT}}$ may not represent the heterogeneity well if the important scale of variability is at a scale smaller than that of the MODIS CTT resolution $(5 \mathrm{~km})$ and thus we also examine the effect of using $\gamma_{\tau}$ and the relationship between $\gamma_{\tau}$ and $\sigma_{\mathrm{CTT}}$.

\subsection{Method for assessing the effect of solar zenith angle on MODIS cloud retrievals}

The operational MODIS Level-3 (hereafter L3) data sets are produced by averaging individual Level-2 (L2) swaths onto a $1^{\circ} \times 1^{\circ}$ grid on a daily basis. MODIS swaths from individual satellites (i.e. Terra or Aqua) start to overlap at latitudes higher than $23^{\circ}$, which means that some locations at such latitudes are sampled on more than one consecutive overpass. At latitudes higher than $62^{\circ}$ three consecutive overpasses are possible and near the poles overpasses occur throughout the day. More than one daylight overpass for a given location means that retrievals are made at more than one local time and therefore with more than one value of $\theta_{0}$.

As an example, Fig. 1a and $b$ show the maximum $\theta_{0}$ of all of the available MODIS Terra (equator crossing time 10:30 LT) and Aqua (13:30 LT) daytime overpasses for 20 June 2007. The results for Terra and Aqua are very similar. Daytime overpasses are defined as $\theta_{0} \leq 81.4^{\circ}$, which is the $\theta_{0}$ range for which optical retrievals are made $\left(\tau, r_{\mathrm{e}}, N_{\mathrm{d}}\right.$, etc.). At high southern latitudes there are no data because $\theta_{0}$ never reaches below this value on this austral mid-winter day. The individual swaths, with data gaps in between at low latitudes, can be discerned from this figure. It also demonstrates the variety of maximum $\theta_{0}$ values at a given latitude due to the differing number of orbit overlaps that are possible. At low latitudes lower $\theta_{0}$ values are present towards the eastern (western) regions of the swaths for Terra (Aqua) since these off-nadir regions are sampled at later (earlier) local times, which are closer to noon relative to the western (eastern) parts of the swaths.

At high northern latitudes the pattern becomes more complicated due to there being several overpasses per day with $\theta_{0}<81.4^{\circ}$. The exact number varies with longitude as well as latitude, since it depends on how many of the swaths overlap. Figure 1c and d show the difference between the maximum and minimum $\theta_{0}$ for the same day. From this pattern the changes in the number of overpasses per day can be discerned. North of $62^{\circ} \mathrm{N}$ the maximum minus minimum $\theta_{0}$ can reach between $\sim 20-45^{\circ}$ showing that even though the maximum $\theta_{0}$ is high there will be some overpasses with a more reasonable $\theta_{0}$ akin to those sampled at much lower latitudes. The pattern changes from day to day as the centres of the swath paths precess to different longitudes over a 16-day period.

For high-latitude regions very high $\theta_{0}$ retrievals are made. Data from all available overpasses are averaged into a daily value for the Level-3 product, which gives the potential for the inclusion of very high $\theta_{0}$ retrievals and may lead to biases in the retrieved $\tau, r_{\mathrm{e}}$ and $N_{\mathrm{d}}$ values, for the reasons discussed earlier. However, the effect of $\theta_{0}$ on $r_{\mathrm{e}}$ and $N_{\mathrm{d}}$ retrievals remains unquantified and a demonstration of the effect of using actual MODIS data is also lacking. Here we make such an estimate.

\subsubsection{MODIS data employed}

Determining the effect of $\theta_{0}$ on MODIS retrievals using the MODIS data record without also aliasing change in other variables is difficult. At latitudes lower than around $62^{\circ}$ there are a maximum of two overpasses in daylight hours for each satellite and thus relatively little $\theta_{0}$ range is sampled during one day for a given location. To test a wide range of $\theta_{0}$ for lower latitudes therefore requires that either a long time period is sampled in order to incorporate seasonal changes in $\theta_{0}$, or that a range of latitudes is sampled. Unfortunately both of these are likely to also cause systematic (but unquantified) changes in $N_{\mathrm{d}}$ due to real-world (i.e. non-retrieval based) changes.

Sampling at higher latitudes, however, offers a solution, although there are limitations there too. Because Aqua and Terra are polar orbiters, at a high enough latitude there will be overpasses throughout the day, which will encompass a wide range of $\theta_{0}$ values. Unfortunately, throughout most of the year the Sun is too low in the sky to get a low enough minimum $\theta_{0}$ to allow a wide range of $\theta_{0}$ values to be tested. 

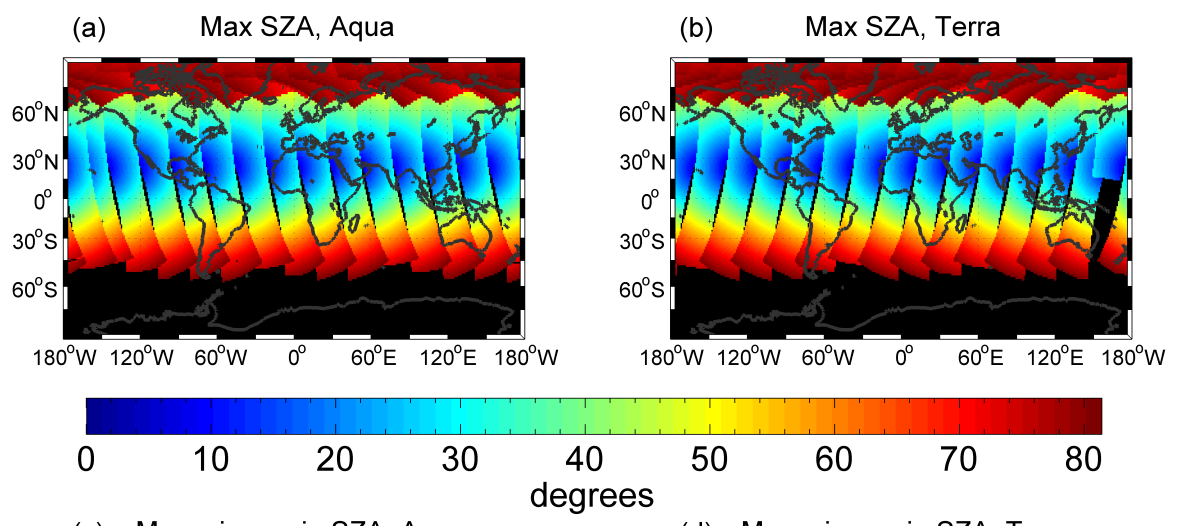

(c) Max minus min SZA, Aqua

(d) Max minus min SZA, Terra

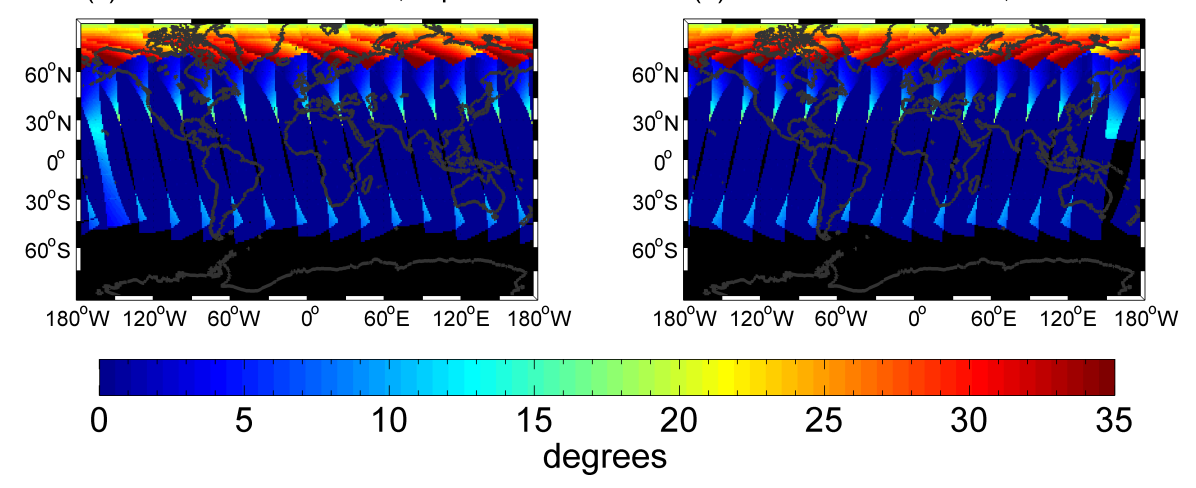

Figure 1. Solar zenith angle (SZA, or $\theta_{0}$ ) properties for a single day (20 June 2007; approximately the solstice) of maximum $\theta_{0}$ (a and b) and maximum minus minimum $\theta_{0}(\mathbf{c}$ and $\mathbf{d})$ for daytime $\left(\mathrm{SZA} \leq 81.4^{\circ}\right)$ data. (a and $\left.\mathbf{c}\right)$ MODIS Aqua, (b and d) MODIS Terra.

However, at mid-summer it is possible to achieve minimum $\theta_{0}$ values as low as $45^{\circ}$ at latitudes as high as $70^{\circ}$ and thus a reasonable range of $\theta_{0}$ can be sampled.

A problem with high latitudes, though, is the presence of ice-covered surfaces. Retrievals over ice are generally considered problematic (King et al., 2004) and it is possible that this would introduce its own biases. The Antarctic continent covers most longitudes at the relevant latitudes in the Southern Hemisphere and in regions where that is not the case there is sea ice present in mid-summer. However, in the Northern Hemisphere the Barents and Norwegian seas are relatively sea-ice-free for most of the year (Fig. 2) and it is here (in the boxed region of the figure) that we focus our efforts.

The period of 13-30 June was chosen for this study in order to allow for a full cycle of the 16-day orbital path precession of the Aqua and Terra satellites and to allow a variety of solar and viewing zenith angle combinations for a given location. However, the period is likely short enough that there would be little seasonal variation in the daily mean $\theta_{0}$, which is also aided by the choice of a mid-summer time period. Seasonal changes are much smaller than the changes in $\theta_{0}$ due to the diurnal sampling by MODIS. This period is analysed for the years 2007-2010 for both the Aqua and Terra satellites.
When trying to discern the effects of $\theta_{0}$ on $N_{\mathrm{d}}$ it is important to sample only a small range of latitudes since $\theta_{0}$ is a strong function of latitude and $N_{\mathrm{d}}$ also may systematically change with latitude. Therefore this would produce spurious results. Thus, the box shown in Fig. 2 was chosen to cover a small latitude range of only $72-75^{\circ} \mathrm{N}$. A fairly large longitude range ( -3 to $48^{\circ} \mathrm{E}$ ) is chosen to give lower statistical noise. $\theta_{0}$ values for MODIS overpasses do not vary systematically with longitude and so regional cloud properties should not introduce any apparent $\theta_{0}$ effects. In order to assess potential longitude-dependent or regional effects, we have investigated the effect of splitting the domain into equally sized eastern and western regions and found that the results are very similar for both regions. Also, similar results are obtained for both the first half and the second half of the time period. VZA and $\phi$ can both co-vary with $\theta_{0}$ and certain ranges of both are known to introduce biases in MODIS cloud optical property retrievals as discussed in Sect. 2.1. However, we will show shortly that it is possible to isolate the effects of $\theta_{0}$ and VZA.

Apart from the effects just mentioned, the only remaining likely source of systematic variation in cloud properties with $\theta_{0}$ (apart from the unidentified radiative sources that lead to retrieval errors that we are looking for) is that due to diurnal 

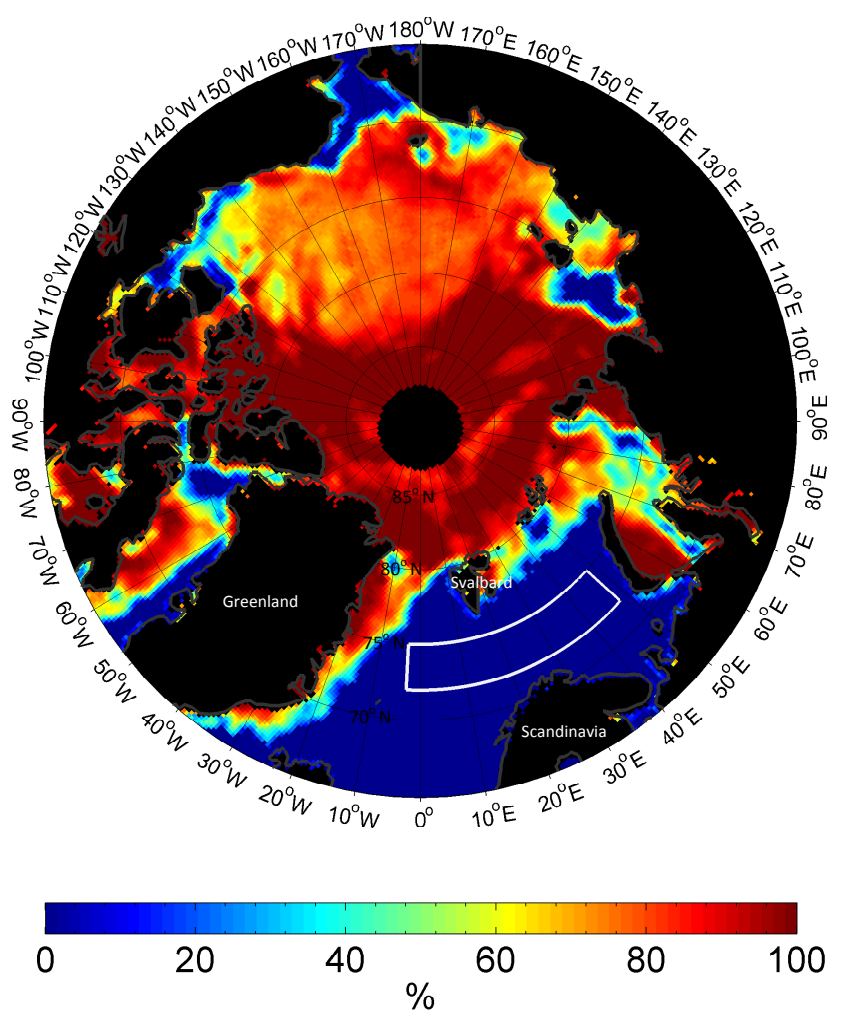

Figure 2. The region of interest for this study (white box; 72 to $75^{\circ} \mathrm{N},-3$ to $48^{\circ} \mathrm{E}$ ) plotted onto a map of sea-ice areal coverage (\%) for 13 June 2007, which was the start of the studied period. Sea ice generally was diminishing with time throughout the period.

variation. Since we are utilizing the diurnal variation in $\theta_{0}$ we cannot remove any potential artifacts due to this. However, we argue that the effect of the diurnal cycle on our results is likely to be small. For brevity, a detailed discussion of this issue is deferred to Appendix B.

\subsubsection{Methodology for the MODIS data processing}

In a similar manner to that used to create the MODIS L3 product (King et al., 1997; Oreopoulos, 2005), we processed MODIS collection 5.1 joint-L2 swaths into $1^{\circ} \times 1^{\circ}$ grid boxes. Joint-L2 data is a subsampled version of the full L2 swaths (sampling every 5 th $1 \mathrm{~km}$ pixel) that also contains fewer parameters. To confirm that there is no effect from the subsampling inherent in the joint-L2 product, we also performed the analysis using the standard L2 files for only one of the years and found little change to the results, consistent with Oreopoulos (2005).

Unless otherwise mentioned, for the MODIS data set referred to throughout the rest of this paper we have applied some restrictions to each $1^{\circ} \times 1^{\circ}$ grid box in order to attempt to remove artifacts that may cause biases:
1. At least 50 joint-L2 $1 \mathrm{~km}$ resolution pixels from the MODIS swath were required to have been sampled within each grid box. This represents approximately a third of the total possible for grid boxes at these latitudes.

2. At least $90 \%$ of the available pixels were successfully designated as either liquid cloud, ice cloud, undetermined cloud, or as clear by the MODIS operational optical cloud properties retrieval algorithm (using the "primary cloud retrieval phase outcome" flag) and did not suffer from sunglint. For the other $10 \%$ of pixels there was either sunglint, or the MODIS algorithm could not set them as clear or cloudy, which could be due to various factors. Analysis was not performed on such pixels.

3. All of the pixels remaining after the above restriction were required to be of liquid phase based upon the "primary cloud retrieval phase flag". Thus the liquid cloud fraction over the grid box $\left(\mathrm{CF}_{\text {liq }}\right)$ was at least $90 \%$. A high cloud fraction helps to ensure that the clouds are not broken (except for the possibility of clear regions in the $10 \%$ mentioned above and subpixel clear regions), since broken clouds are known to cause biases in retrieved optical properties due to photon scattering through the sides of clouds. Often retrievals of droplet concentrations, which rely on optical depth and effective radius, are restricted to high cloud fraction fields for this reason (B07; PZ11) and so we focus on such data points here. However, an overcast grid box still allows cloud heterogeneities caused by variations in cloud top height, cloud optical extinction (including subpixelscale holes), cloud depth, etc. Thus homogeneity is not ensured. Such issues are discussed in detail in Sect. 2.

4. It was required that for at least $90 \%$ of the pixels remaining after the previous restriction the "cloud mask status" indicated that the cloud mask could be determined, the "cloud mask cloudiness flag" was set to "confident cloudy", successful simultaneous retrievals of both $\tau$ and $r_{\mathrm{e}}$ were performed and the cloud water path confidence from the MODIS L2 quality flags was designated as "very good confidence" (the highest level possible). This is a little different from the official MODIS L3 product where a set of cloud products are provided that are weighted using the Quality Assurance (QA) flags. Rather than weighting our L3-like product with the QA flags we have simply restricted our analysis to pixels with the highest confidence for water path.

5. The mean CTT is restricted to values warmer than $268 \mathrm{~K}$. This is done both to avoid clouds containing ice and because there appear to be problems in identifying the phase of clouds at high $\theta_{0}$ for temperatures colder than this. These points are discussed in what follows. 
(a)
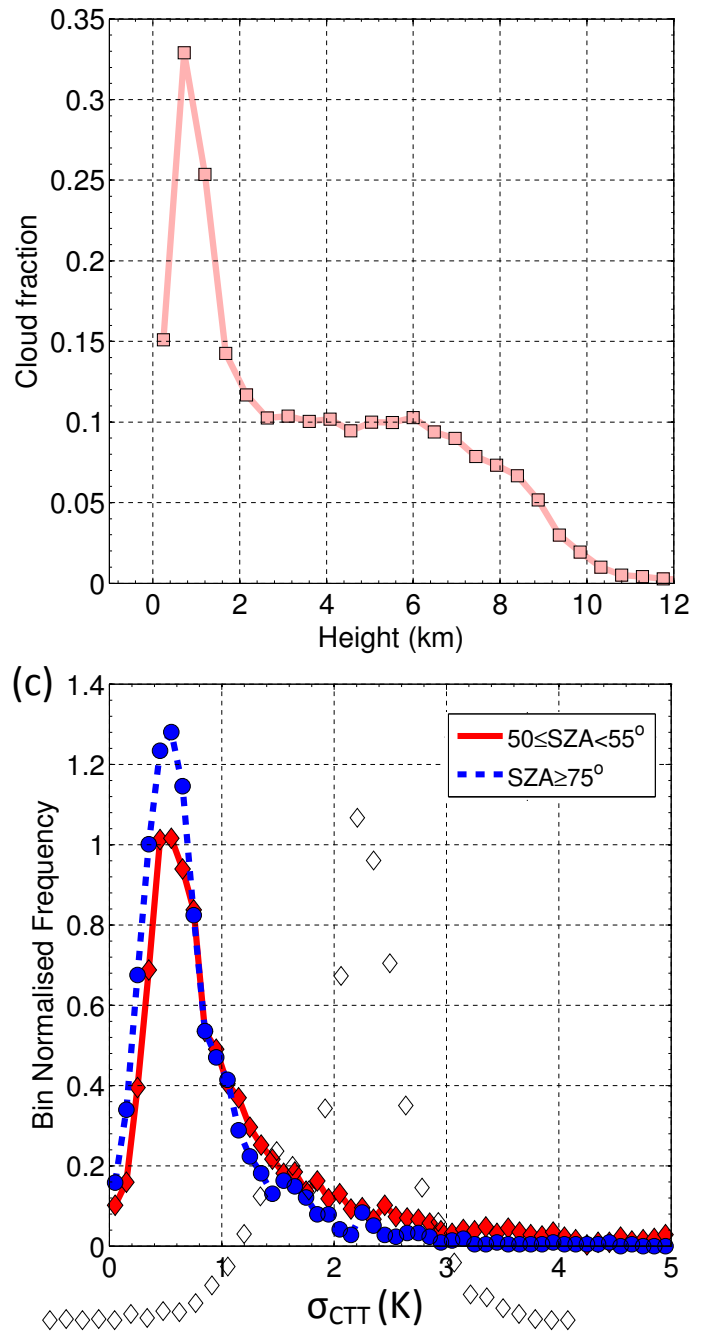

(b)

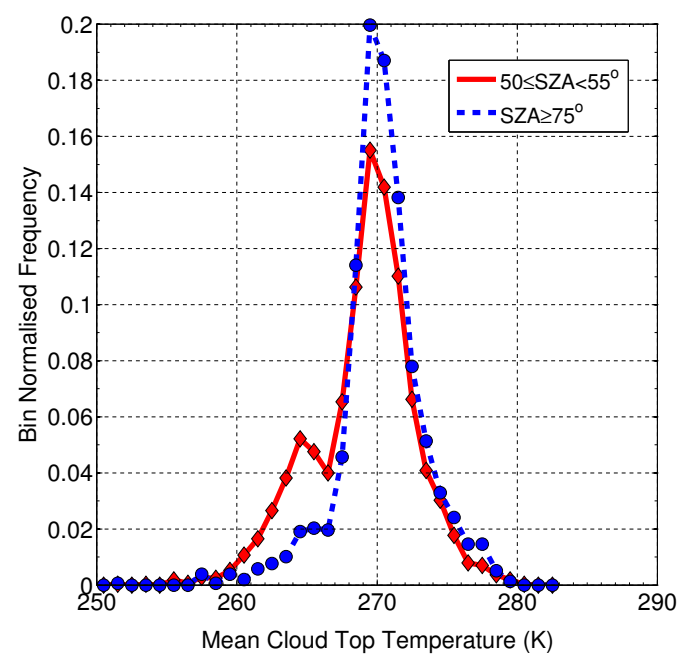

Figure 3. (a) CALIPSO cloud fraction vs. height for the month of June for the years 2007-2010. (b) PDFs of mean MODIS grid box cloud top temperature (CTT) for grid boxes containing liquid clouds only (restrictions $1-4$ applied, see text) and for VZA $\leq 41.4^{\circ}$. The difference between the low and high $\theta_{0}$ PDFs is highly likely to be due to phase determination problems at high $\theta_{0}$ (see text for explanation). When considering clouds of all phases the PDFs are identical (not shown). (c) PDFs of the standard deviation of CTT within grid boxes for data points that have had restrictions $1-5$ applied (see text) and for VZA $\leq 41.4^{\circ}$. All plots are for the region highlighted in Fig. 2.

The restriction to high $\mathrm{CF}_{\text {liq }}$ also serves the purpose of attempting to ensure that grid boxes with a significant ice cloud fraction are not sampled. However, since MODIS is likely to determine the phase of only the upper regions of the cloud it is possible that clouds contain ice in their lower regions. Indeed, it has been observed from ground-based measurements that Arctic clouds can be dominated by liquid in their upper regions whilst precipitating snow (Morrison et al., 2012). Whether such clouds would be identified by MODIS as being liquid or ice and whether the presence of ice lower in the cloud would affect MODIS retrievals of $\tau, r_{\mathrm{e}}$ and $N_{\mathrm{d}}$ are open questions. However, the presence of ice is by no means guaranteed, especially at temperatures closer to $0{ }^{\circ} \mathrm{C}$ where ice nuclei concentrations are likely to be very low (DeMott et al., 2010). Ground measurements show that at temperatures warmer than around $-5^{\circ} \mathrm{C}$ Arctic stratus clouds can, depending on location, be almost completely dominated by liquid (de Boer et al., 2011). Issues regarding the likelihood of the presence of ice are discussed further in Appendix C.

\section{Results}

\subsection{Examining the properties of the sampled clouds}

We first examine the distribution of cloud fraction vs. height within the specified region using the CALIPSO GOCCPv2.1 (Chepfer et al., 2010) data set. (Fig. 3). These data are for the month of June for the period 2007-2010. Here it needs to 
be borne in mind that CALIPSO can observe clouds at multiple heights within one profile, although thick clouds will rapidly attenuate the signal. CALIPSO reveals the presence of a significant number of high-level clouds although the mode height is $0.48-0.96 \mathrm{~km}$. Thus there are a lot of clouds that likely reside within the boundary layer and which would therefore be well suited to the application of the $N_{\mathrm{d}}$ estimate using MODIS, as described in Sect. 3.1 and Appendix A.

Figure $3 \mathrm{~b}$ shows Probability Density Function (PDFs) of MODIS grid box mean CTT for low and high $\theta_{0}$ cases for grid boxes with restrictions 1-4 applied. The PDFs reveal that for both low and high $\theta_{0}$ almost all of these data points have CTTs warmer than $260 \mathrm{~K}$ with a mode at around $269 \mathrm{~K}$. Thus, the majority of the clouds have subzero CTTs, which may allow for some ice formation. However, as discussed above and in Appendix C, ground-based observations in the Arctic generally indicate a dominance of liquid or mixed phase clouds for such cloud top temperatures.

For the low $\theta_{0}$ data there is an interesting secondary mode at around $264 \mathrm{~K}$, which is not present for high $\theta_{0}$. Although this could indicate physical differences between the low and high $\theta_{0}$ clouds (e.g. fewer liquid cloud tops at high $\theta_{0}$ at the colder temperatures, perhaps related to a reduction in cloud top SW heating), the difference seems more likely to be due to retrieval differences, since if restrictions 3 and 4 are lifted the low and high $\theta_{0}$ CTT PDFs are almost identical (not shown). This indicates a difference between the number of pixels that are determined to be liquid at low and high $\theta_{0}$, despite having the same CTT distribution for the general unscreened population. A change in cloud glaciation due to a reduction in SW heating at high $\theta_{0}$ might be expected to be accompanied by changes in CTT. Further, there are more pixels classified as "undetermined" phase at high $\theta_{0}$, which also points towards problems with the phase determination being the cause. The limiting of CTT to values warmer than $268 \mathrm{~K}$ (restriction 5 above) thus circumvents these issues regarding the differences in CTT PDFs at CTT $<\sim 268 \mathrm{~K}$ and those due to the increased likelihood of ice at such temperatures.

Figure $3 \mathrm{c}$ shows PDFs of $\sigma_{\mathrm{CTT}}$ for grid boxes that have had restrictions $1-5$ applied and for $\mathrm{VZA} \leq 41.4^{\circ}$ for both low and high $\theta_{0}$. The distributions at low and high $\theta_{0}$ are very similar suggesting that the variability of cloud top temperature is comparable when the Sun is oblique (i.e. near sunrise/sunset) and when it is higher in the sky (near local noon), at least for this restricted subset of clouds and at the scales probed by the $5 \mathrm{~km}$ resolution CTT measurements. This, along with the similar shapes of the CTT distribution for CTT $<\sim 268 \mathrm{~K}$ (Fig. 3b) indicates that the diurnal cycle is having little impact on the physical nature of clouds. Therefore we might expect that the subsets of clouds at low and high $\theta_{0}$ are likely to be physically similar, so that any differences in the retrieved $\tau$ and $r_{\mathrm{e}}$ are primarily due to retrieval artifacts.

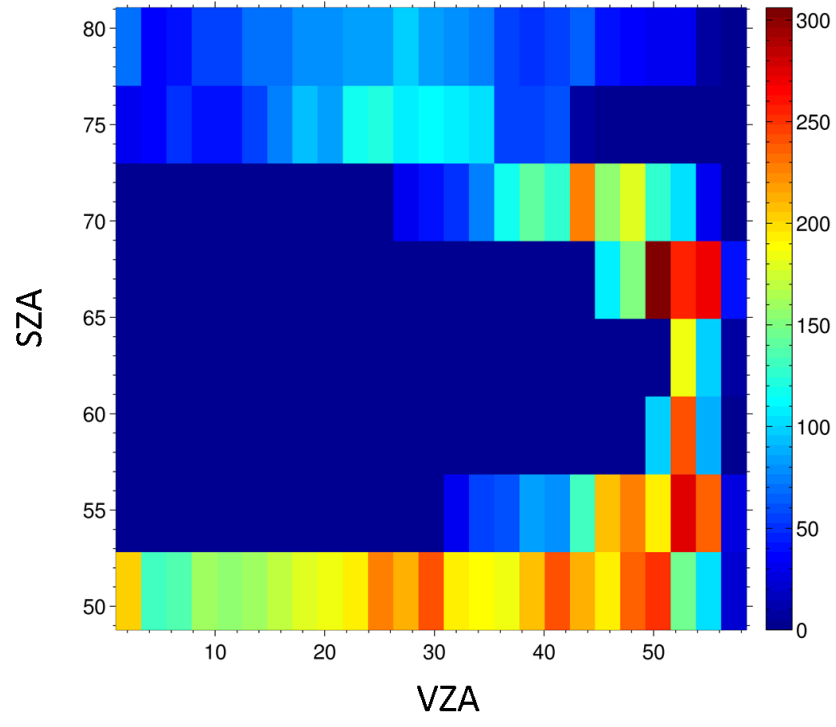

Figure 4. 2-D histogram of solar zenith angle (SZA) vs. viewing zenith angle (VZA) for the $1^{\circ} \times 1^{\circ}$ grid boxes used as data points in this study. The colours represent the number of such data points at each pairing. Data have been filtered according to the criteria outlined in the text.

Figure 4 shows the number of $1^{\circ} \times 1^{\circ}$ data points for each pairing of viewing (VZA) and solar zenith angles (SZA, or $\theta_{0}$ ) for the data set following the application of restrictions $1-5$. The figure reveals that between $\theta_{0}$ of $\sim 55^{\circ}$ and $67^{\circ}$ there is a only a narrow range of VZA encompassing only values $>50^{\circ}$. For $\theta_{0}<52.5^{\circ}$ and $\theta_{0}>72.5^{\circ}$ a spread across almost all possible VZA values is sampled. This will allow the testing of the $\theta_{0}$ effect in isolation of potential effects due to a high VZA. It also shows that restricting the maximum VZA of MODIS L3 data points would not be enough to avoid all high $\theta_{0}$ data being included. The sampled $\phi$ (not shown) all correspond to angles within $30^{\circ}$ of side scattering $\left(=90^{\circ}\right)$, comprising two narrow ranges: $65-72.5^{\circ}$ and $112.5-120^{\circ}$. Thus, the variability of $\phi$ is unlikely to greatly affect the results.

\subsection{Cloud properties vs. $\theta_{0}$}

We now show results of averages over the whole domain and time period of various retrieved microphysical cloud properties in different SZA bins. The results are split into averages for data in which VZA was $\leq 41.4^{\circ}$ and for $>41.4^{\circ}$ to isolate the effects of $\theta_{0}$ from those of VZA. The angle $41.4^{\circ}$ is chosen since this represents the halfway point of $\cos (\theta)$ between $0^{\circ}$ and the maximum MODIS VZA of $60^{\circ}$. It has also been shown that a higher VZA results in an increase in the reported MODIS cloud fraction (Maddux et al., 2010). This was thought to have been due to lower instrument resolution and an increased path length between the scene and 
the satellite with increasing VZA, both of which make cloud detection more likely.

In subsequent plots, error bars represent the combined (in quadrature) instrument and sampling errors. L2 MODIS uncertainties in $\tau$ and $r_{\mathrm{e}}$ (as provided with the retrievals) are averaged (using a simple mean) to produce $1^{\circ} \times 1^{\circ}$ uncertainties. This therefore assumes that L2 pixel uncertainties are fully correlated within each L3 box $\left(1^{\circ} \times 1^{\circ}\right)$, which is also the case for the operational L3 uncertainty estimate. However, when calculating the domain and period mean values from $1^{\circ} \times 1^{\circ}$ boxes the instrument uncertainties are combined in quadrature assuming no correlation in order to assess the magnitude of random instrument errors. Departures outside of the calculated error range are therefore likely to represent a systematic bias. Sampling errors are calculated based on the standard deviation of the quantity of interest and the number of samples within each bin.

\subsubsection{Optical depth}

Figure 5 shows the mean optical depth in each SZA bin. At intermediate $\theta_{0}$, only VZA $>41.4^{\circ}$ data are available and for the $\theta_{0}$ bin centred near to $75^{\circ}$ only data for $\mathrm{VZA}<41.4^{\circ}$ are available. This is due to the sampling pattern of MODIS (as demonstrated in Fig. 4).

Mean $\tau$ values are very similar for the two VZA ranges at both low $\theta_{0}$, and for the $\theta_{0}$ bin centred around $\sim 71^{\circ}$. For the $79.1^{\circ}$ bin the $\tau$ value for high VZA is $14 \%$ larger than that for low VZA. Although the error associated with the high VZA value in this $\theta_{0}$ bin is fairly large, this might indicate a dependence of $\tau$ on VZA at very high $\theta_{0}$, although it is also possible that the tendency to observe a higher cloud fraction at high VZA could also be having an influence on the identification of scenes with cloud fraction $>90 \%$. It is conceivable that the misdiagnosis of lower cloud fraction scenes as overcast might affect higher $\theta_{0}$ retrievals more strongly than low $\theta_{0}$ ones due to a stronger influence of cloud heterogeneity at high $\theta_{0}$. Heterogeneity effects are discussed in more detail in Sect. 4.4.

The high VZA results show that $\tau$ is fairly constant up to a $\theta_{0}$ value of approximately $65-70^{\circ}$. It is speculated that this would also have been the case for low VZA retrievals if they had been made. For both low and high VZA the $\tau$ values at the highest $\theta_{0}$ are higher than those at the lowest $\theta_{0}$. The relative increases in $\tau$ between the lowest and highest $\theta_{0}$ bins were 70 and $92 \%$ for the low and high VZA cases, respectively, representing very large increases in $\tau$ due to increasing $\theta_{0}$. Table 1 lists these percentage increases for $\tau, r_{\mathrm{e}}$ and $N_{\mathrm{d}}$ both for these cases and for some other scenarios that will be discussed later. Figure 6 shows PDFs of $\tau$ at low $\left(50-55^{\circ}\right)$ and high $\left(75-81.4^{\circ}\right) \theta_{0}$ ranges for low VZA only. The distribution shapes are approximately lognormal in both cases and are essentially just shifted towards higher values in the high $\theta_{0}$ case; Table 2 gives the mean $\tau$ values and the normalized standard deviations.

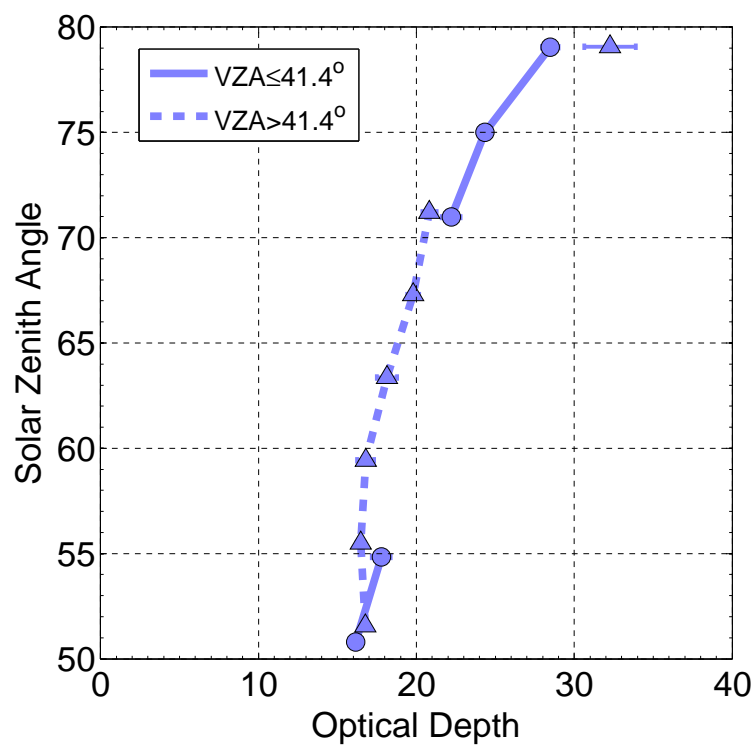

Figure 5. Mean optical depth vs. solar zenith angle for different ranges of viewing zenith angle (VZA, see legend). The errors are discussed in the text and are represented by horizontal error bars. In most cases they are too small to be visible.

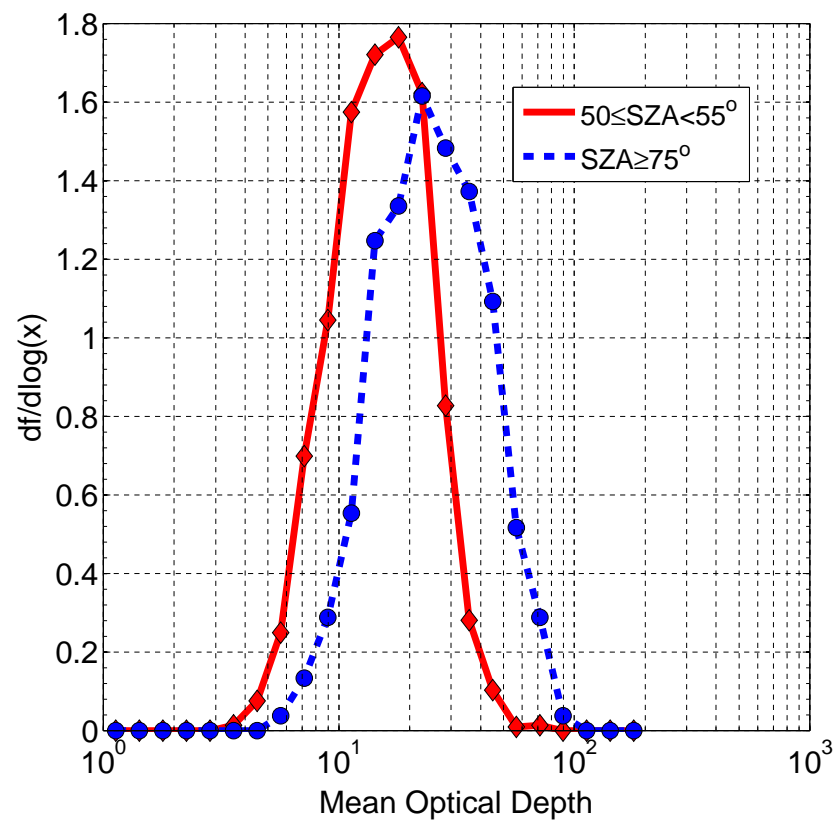

Figure 6. PDFs of optical depth $(\tau)$ for low viewing zenith angles $($ VZA $\leq 41.4)$ and for different solar zenith angles (SZA or $\theta_{0}$, see the legend). Other data restrictions are described in the text. Probabilities are normalized by the bin widths in $\log _{10}$ space.

\subsubsection{Effective radius}

For $r_{\mathrm{e}}$ the results are more complicated (Fig. 7a). Here results from the three different MODIS retrieval wavelengths for $r_{\mathrm{e}}$ are shown $\left(r_{\mathrm{e} 1.6}, r_{\mathrm{e} 2.1}\right.$ and $\left.r_{\mathrm{e} 3.7}\right)$. The standard MODIS 
Table 1. Changes in various quantities between the lowest and highest $\theta_{0}$ bins (high minus low). $\Delta N_{\Delta \tau}$ and $\Delta N_{\Delta \mathrm{re}}$ are the changes in $N_{\mathrm{d}}$ from paired $\tau$ and $r_{\mathrm{e}}$ samples constructed using Latin Hypercube Sampling (see Appendix D) for, respectively, separate changes in $\tau$ and $r_{\mathrm{e}}$ (see text). Four different data subsets are shown for which various restrictions have been applied: Low VZA: VZA $\leq 41.4^{\circ}$; high VZA: VZA $>41.4^{\circ}$; low $\sigma_{\mathrm{CTT}}: \sigma_{\mathrm{CTT}} \leq 0.65 \mathrm{~K}$; high $\sigma_{\mathrm{CTT}}: \sigma_{\mathrm{CTT}}>1 \mathrm{~K}$. For "All $\sigma_{\mathrm{CTT}}$ " there were no restrictions on $\sigma_{\mathrm{CTT}}$.

\begin{tabular}{|c|c|c|c|c|c|c|c|c|c|c|c|c|c|}
\hline \multirow[b]{2}{*}{ Data subset } & \multirow[b]{2}{*}{$\begin{array}{l}\Delta \tau \\
(\%)\end{array}$} & \multicolumn{4}{|c|}{$2.1 \mu \mathrm{m}$} & \multicolumn{4}{|c|}{$1.6 \mu \mathrm{m}$} & \multicolumn{4}{|c|}{$3.7 \mu \mathrm{m}$} \\
\hline & & $\begin{array}{l}\Delta N_{\mathrm{d}} \\
(\%)\end{array}$ & $\begin{array}{l}\Delta r_{\mathrm{e}} \\
(\%)\end{array}$ & $\begin{array}{l}\Delta N_{\Delta \tau} \\
(\%)\end{array}$ & $\begin{array}{l}\Delta N_{\Delta \mathrm{re}} \\
(\%)\end{array}$ & $\begin{array}{l}\Delta N_{\mathrm{d}} \\
(\%)\end{array}$ & $\begin{array}{l}\Delta r_{\mathrm{e}} \\
(\%)\end{array}$ & $\begin{array}{l}\Delta N_{\Delta \tau} \\
(\%)\end{array}$ & $\begin{array}{l}\Delta N_{\Delta \mathrm{re}} \\
(\%)\end{array}$ & $\begin{array}{l}\Delta N_{\mathrm{d}} \\
(\%)\end{array}$ & $\begin{array}{l}\Delta r_{\mathrm{e}} \\
(\%)\end{array}$ & $\begin{array}{l}\Delta N_{\Delta \tau} \\
(\%)\end{array}$ & $\begin{array}{l}\Delta N_{\Delta \mathrm{re}} \\
(\%)\end{array}$ \\
\hline $\begin{array}{l}\text { All } \sigma_{\mathrm{CTT}} \\
\text { low VZA }\end{array}$ & 69.8 & 48.3 & -4.8 & 29.26 & 14.24 & 39.1 & -1.1 & 29.20 & 4.99 & 51.2 & -7.4 & 29.27 & 20.34 \\
\hline $\begin{array}{l}\text { All } \sigma_{\mathrm{CTT}}, \\
\text { high VZA }\end{array}$ & 92.4 & 65.3 & -8.0 & 37.36 & 23.83 & 46.8 & -1.6 & 37.34 & 4.67 & 67.9 & -8.7 & 37.28 & 30.14 \\
\hline $\begin{array}{l}\text { Low } \sigma_{\mathrm{CTT}} \\
\text { low VZA }\end{array}$ & 79.1 & 55.2 & -5.7 & 32.54 & 15.86 & 45.8 & -2.2 & 32.58 & 7.46 & 65.8 & -9.5 & 32.55 & 27.21 \\
\hline $\begin{array}{l}\text { High } \sigma_{\mathrm{CTT}} \text {, } \\
\text { low VZA }\end{array}$ & 49.7 & 56.4 & -8.0 & 22.25 & 26.10 & 36.9 & -2.1 & 22.28 & 9.13 & 61.3 & -10.7 & 22.32 & 34.45 \\
\hline
\end{tabular}

(a)

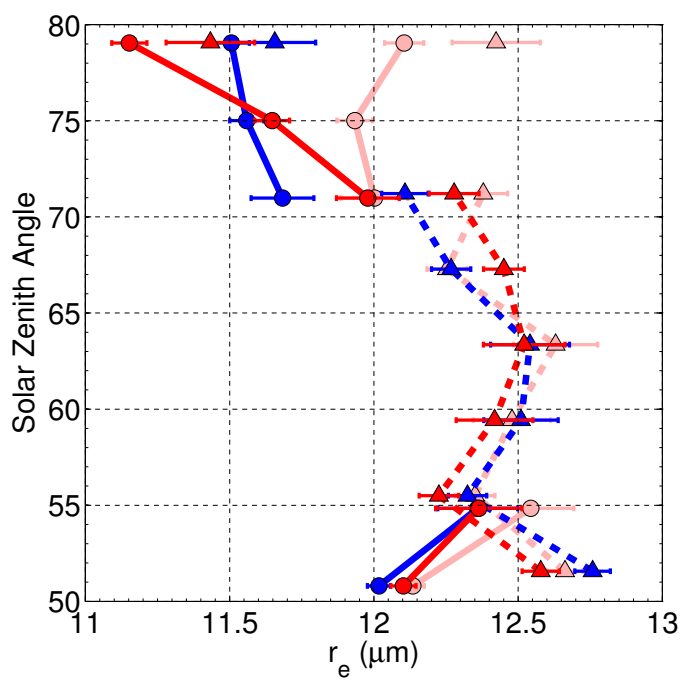

(b)

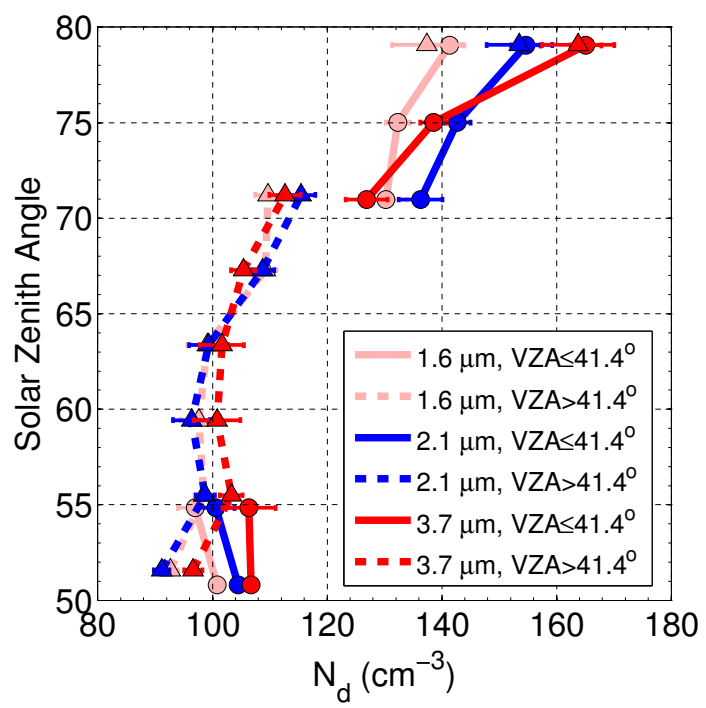

Figure 7. As for Fig. 5 except for the mean effective radius (a) and droplet concentration (b) for the different MODIS bands.

wavelength is $2.1 \mu \mathrm{m}$ and $r_{\mathrm{e}}$ errors are only available for this band. Therefore, the percentage errors for this wavelength are applied as errors for the other wavelengths to give an estimate of the expected uncertainty.

For the 2.1 and $3.7 \mu \mathrm{m}$ bands there is a decrease in the mean $r_{\mathrm{e}}$ between the lowest and highest $\theta_{0}$ bins. This is also evident in the PDFs in Fig. 8 (low VZA only), which show a shape close to a normal distribution. The individual changes between the two ranges of $50-55^{\circ}$ and $75-81.4^{\circ}$ are listed in Table 1 for both low and high VZA; for $r_{\mathrm{e} 2.1}$ there is a mean decrease of $5 \%$ for low VZA and $8 \%$ for high VZA, whereas for $r_{\mathrm{e} 3.7}$ the corresponding decreases are $7.4 \%$ and $8.7 \%$. The decreases are much smaller in magnitude for the $1.6 \mu \mathrm{m}$ band, being only 1.1 and $1.6 \%$ for the low and high VZA ranges, respectively. Thus in all cases there is a slightly larger decrease at high VZA than at low VZA. The high VZA results span the $\theta_{0}$ range between $51.5^{\circ}$ and $71.5^{\circ}$ and suggest a lack of dependence on $\theta_{0}$ in this range, which is similar to the $\tau$ result.

It is also worth mentioning that at both low and high $\theta_{0}$ the observed $r_{\mathrm{e}}$ values were higher for the upper range of VZA than for the lower range. This is interesting because an increase in $r_{\mathrm{e}}$ with VZA was also observed in Maddux et al. (2010, see their Fig. 2).

For a given VZA range there is generally very good agreement between $r_{\mathrm{e} 1.6}, r_{\mathrm{e} 2.1}$ and $r_{\mathrm{e} 3.7}$ for the lower $\theta_{0}$ values. At $\theta_{0}$ of $\sim 71^{\circ}$ and above, the spread between the different $r_{\mathrm{e}}$ values increases with the largest spread being at the highest $\theta_{0}$ value tested. At this $\theta_{0}, r_{\mathrm{e} 3.7}<r_{\mathrm{e} 2.1}<r_{\mathrm{e} 1.6}$ for a given VZA range. This is the opposite of what would be expected from a cloud in which the LWC was increasing with height adiabatically given the different penetration depths of the 

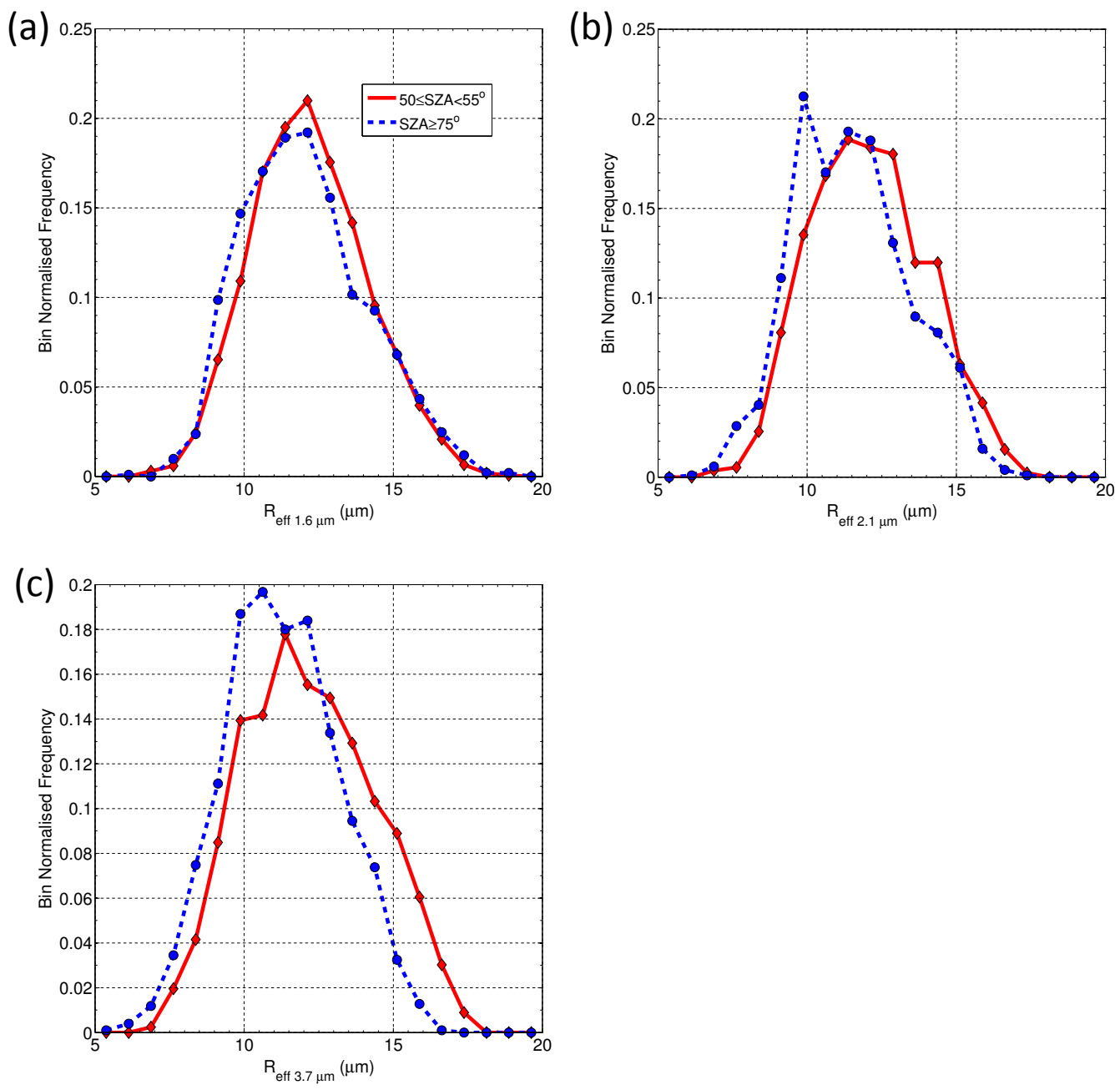

Figure 8. As for Fig. 6 and for $r_{\mathrm{e}}$ and that the probabilities are normalized by the bin widths in linear space. Results are shown for the three different MODIS bands.

different light wavelengths (Platnick, 2000). However, this is in concurrence with several other works that have investigated MODIS retrievals such as Zhang and Plantnick (2011) and Seethala and Horvath (2010). Discussion on the possible reasons for this is deferred to Sect. 5.3.

\subsubsection{Droplet concentration}

Droplet concentrations calculated from the $1^{\circ} \times 1^{\circ}$ mean $\tau$ and mean $r_{\mathrm{e}}$ using Eq. (A1) are shown in Fig. 7b as a function of $\theta_{0}$. The mean $\tau$ and $r_{\mathrm{e}}$ are used rather than the individual $1 \mathrm{~km}$ values to be consistent with previous estimates that use L3 data and to reduce errors that may be caused by high-resolution point estimates. For both the 2.1 and $3.7 \mu \mathrm{m}$ bands mean $r_{\mathrm{e}}$ values were shown to decrease with $\theta_{0}$ and $\tau$ was shown to increase. Therefore, it is perhaps not a surprise that $N_{\mathrm{d}}$ increases with $\theta_{0}$ given Eq. (A1). $N_{\mathrm{d}}$ also increases with $\theta_{0}$ for the $1.6 \mu \mathrm{m}$ band where the $r_{\mathrm{e}}$ decreases were much smaller. This suggests that the increase in $\tau$ is dominating the $N_{\mathrm{d}}$ increase in that case, although it is possible that changes in the spread of the $r_{\mathrm{e}}$ size distribution and/or negative correlation between $\tau$ and $r_{\mathrm{e}}$ could also be playing a role. Issues regarding the relative roles of these factors in causing the changes in $N_{\mathrm{d}}$ with $\theta_{0}$ are discussed in Sect. 4.5.

Figure 9 shows PDFs at low and high $\theta_{0}$ (for low VZA only) and reveals approximately lognormal shapes. For low VZA, the increases in $N_{\mathrm{d}}$ between low and high $\theta_{0}$ were $39 \%, 48 \%$ and $51 \%$ for the 1.6, 2.1 and $3.7 \mu \mathrm{m}$ bands; for high VZA the corresponding increases were $47 \%, 65 \%$ and $68 \%$. In addition, the low VZA values are higher than the high VZA ones for all wavelengths and at all $\theta_{0}$, except for the $3.7 \mu \mathrm{m}$ band at high $\theta_{0}$ where there is a very slight increase with VZA (see Table 2). For the highest $\theta_{0}$ this result is inconsistent with the $\tau$ result whereby higher $\tau$ values occurred for higher VZA. This suggests that the decrease in $r_{\mathrm{e}}$ with increasing VZA is dominating the $N_{\mathrm{d}}$ change with VZA at high $\theta_{0}$. Again, further discussion of such issues is deferred to Sect. 4.5. In a similar manner to $\tau$ and $r_{\mathrm{e}}$ there is a change 
(a)

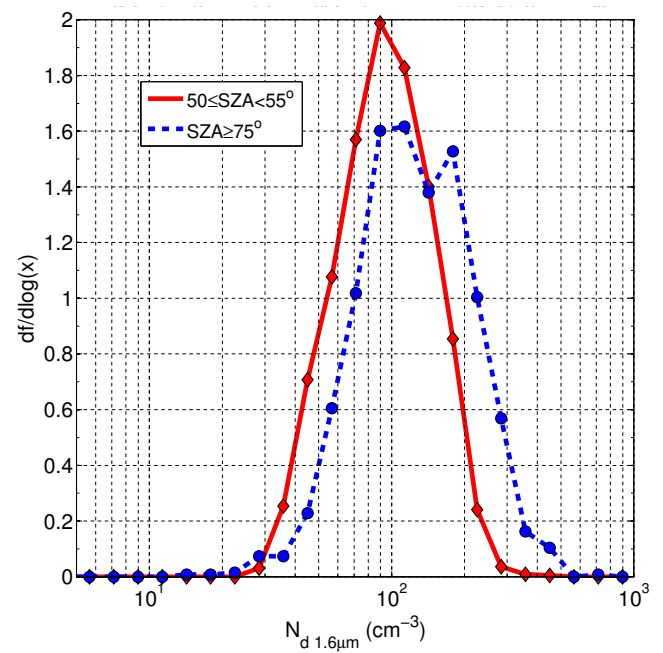

(c)

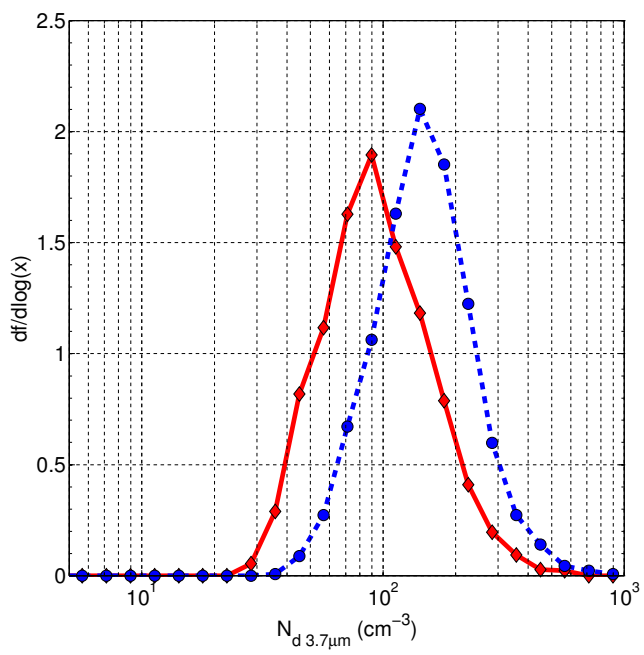

(b)

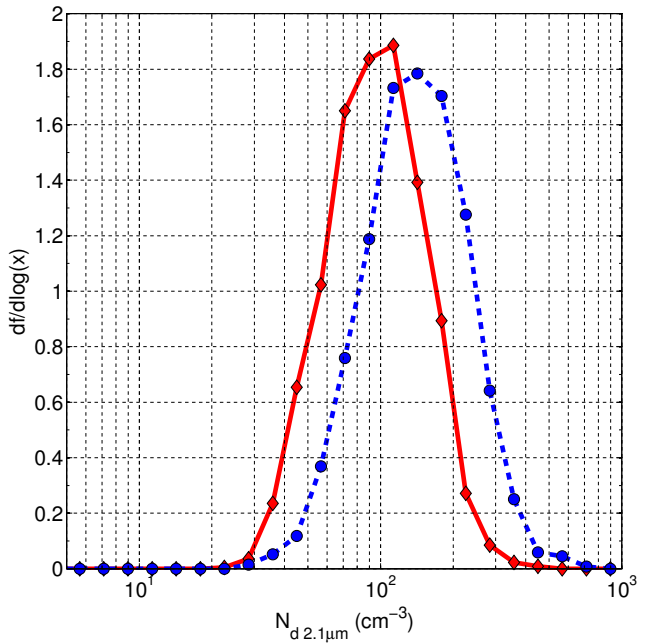

Figure 9. As for Fig. 8 except for $N_{\mathrm{d}}$ except that probabilities are normalized by the bin widths in $\log _{10}$ space.

in the behaviour of $N_{\mathrm{d}}$ at a $\theta_{0}$ value of around $65-70^{\circ}$ with little dependence upon $\theta_{0}$ at lower $\theta_{0}$ values than this.

\subsection{The diurnal cycle}

In Sect. 3.3.1 and Appendix B we discussed the potential of a real (i.e. physical) diurnal cycle of the stratocumulus clouds causing apparent effects due to $\theta_{0}$. Plotting $N_{\mathrm{d}}$ against local time of day (Fig. 10) instead of $\theta_{0}$ indicates that there is very little diurnal cycle in $N_{\mathrm{d}}$ because $N_{\mathrm{d}}$ values are almost constant between the hours of $\sim 7$ and 18:00 LT when using $r_{\mathrm{e} 2.1}$ and $r_{\mathrm{e} 3.7}$. If there was a diurnal cycle in $N_{\mathrm{d}}$ then some variation would be expected. These times correspond to those for which $\theta_{0}$ is $<63-67^{\circ}$, which is consistent with our results in the previous sections that showed $\theta_{0}$ effects for $\theta_{0} \gtrsim 65-70^{\circ}$. The symmetry of the lines around local noon also suggests an effect due to $\theta_{0}$ artifacts rather than a physical diurnal effect since the observed LWP diurnal cycle was shown in O'Dell et al. (2008) to be asymmetrical with a maximum value at around 03:00-06:00 LT.

The results for effective radius (not shown) for $r_{\mathrm{e} 2.1}$ and $r_{\mathrm{e} 3.7}$ are very similar to those of $N_{\mathrm{d}}$. Those for $\tau$ (not shown) do show some asymmetry around local noon that would be consistent with a real diurnal cycle. However, the observed increase in $\tau$ of $70-90 \%$ at high $\theta_{0}$ relative to at low $\theta_{0}$ as described in Sect. 4.2.1 is much larger than the expected 8$17 \%$ increase in $\tau$ due to the LWP diurnal cycle, as calculated from the $<\sim 10-20 \%$ amplitudes of LWP diurnal reported in O'Dell et al. (2008) (see Appendix B for the calculation details).

However, it is difficult to estimate the true $\tau$ diurnal cycle from our results and therefore to fully resolve the effects seen 


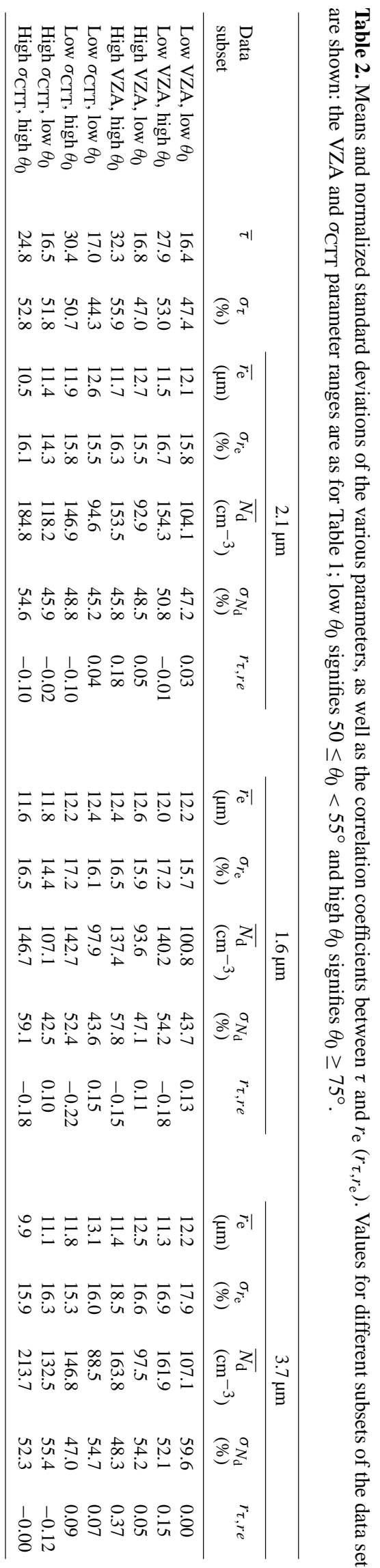

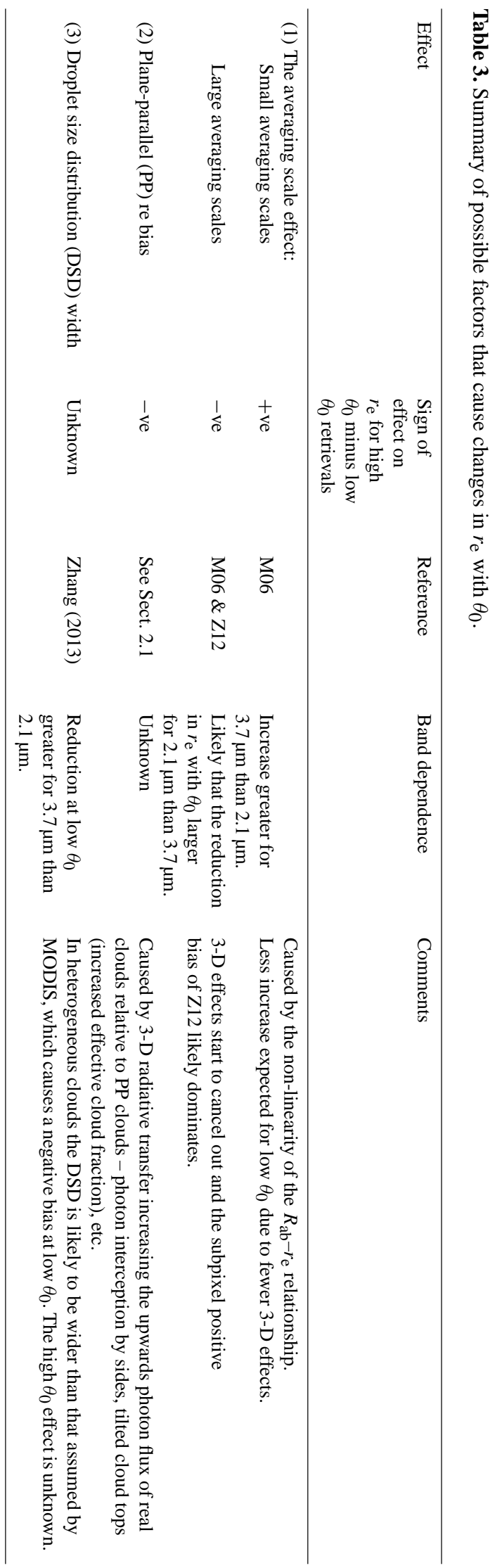



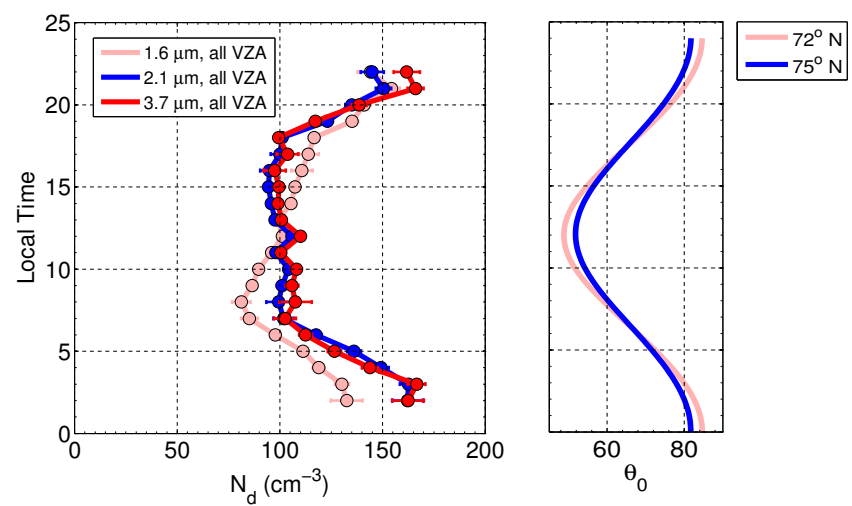

Figure 10. $N_{\mathrm{d}}$ (left panel) and $\theta_{0}$ (right panel) vs. local time of day. For the $r_{\mathrm{e} 2.1}$ and $r_{\mathrm{e} 3.7}$ based $N_{\mathrm{d}}$ retrievals, the flatness of the curves for the times corresponding to lower $\theta_{0}$ suggests that there is little physical diurnal cycle of $N_{\mathrm{d}}$. This suggests that the changes seen at high $\theta_{0}$ are the result of retrieval artifacts and not physical effects. All VZA values are included and $N_{\mathrm{d}}$ values are shown for retrievals made using $r_{\mathrm{e} 1.6}, r_{\mathrm{e} 2.1}$ and $r_{\mathrm{e} 3.7}$. For the $\theta_{0}$ plot, values are shown at the most southern $\left(72^{\circ} \mathrm{N}\right)$ and northern $\left(75^{\circ} \mathrm{N}\right)$ edges of the region. Over the period of study, time variation of $\theta_{0}$ from day to day was very slight for a given local time and grid box.

Table 4. Results from the example calculations of 3-D radiative effects at small averaging scales, as demonstrated in Fig. 17, except that results from various other view angles (VZA) and relative azimuth angles $(\phi)$ are also shown. 3-D effects at high $\theta_{0}$ are assumed to cause an equal increase and decrease $(\Delta R)$ in the reflectances of both the absorbing band $\left(R_{\mathrm{ab}}\right)$ and the non-absorbing band $\left(R_{\mathrm{nab}}\right)$. For this demonstration it is assumed that there are an equal number of small-scale cloud elements all with $r_{\mathrm{e}}=14 \mu \mathrm{m}$ and $\tau=21.4$. Retrievals are then made on the reflectances that have been distorted by the 3 -D effects. The retrievals were made using MODIS lookup tables (LUTs) that are used for converting non-absorbing $(0.86 \mu \mathrm{m})$ and absorbing reflectance pairs into $\tau$ and $r_{\mathrm{e}}$. These are shown for $\theta_{0}=79^{\circ}$ and for the $1.6 \mu \mathrm{m}, 2.1 \mu \mathrm{m}$ and $3.7 \mu \mathrm{m}$ absorbing bands. It can be seen that in all cases the retrieved $r_{\mathrm{e}}$ would be greater than the true $r_{\mathrm{e}}$ of $14 \mu \mathrm{m}$. See Sect. 5.1 for further details.

\begin{tabular}{lllrr}
\hline VZA & $\phi$ & Band & Retrieved $r_{\mathrm{e}}$ & Difference from true $r_{\mathrm{e}}$ \\
\hline $0^{\circ}$ & N/A & $1.6 \mu \mathrm{m}$ & 14.7 & 0.7 \\
$0^{\circ}$ & N/A & $2.1 \mu \mathrm{m}$ & 15.3 & 1.3 \\
$0^{\circ}$ & N/A & $3.7 \mu \mathrm{m}$ & 19.3 & 5.3 \\
& & & & \\
$50^{\circ}$ & $30^{\circ}$ & $1.6 \mu \mathrm{m}$ & 14.1 & 0.1 \\
$50^{\circ}$ & $30^{\circ}$ & $2.1 \mu \mathrm{m}$ & 14.3 & 0.3 \\
$50^{\circ}$ & $30^{\circ}$ & $3.7 \mu \mathrm{m}$ & 14.4 & 0.4 \\
& & & & \\
$50^{\circ}$ & $150^{\circ}$ & $1.6 \mu \mathrm{m}$ & 14.3 & 0.3 \\
$50^{\circ}$ & $150^{\circ}$ & $2.1 \mu \mathrm{m}$ & 14.7 & 0.7 \\
$50^{\circ}$ & $150^{\circ}$ & $3.7 \mu \mathrm{m}$ & 15.5 & 1.5 \\
\hline
\end{tabular}

here into those due to $\theta_{0}$ artifacts and those due to any real diurnal cycle. Therefore, this is left to future work. Another remaining issue is that the diurnal results using $r_{\mathrm{e} 1.6}$ were more complicated than those of $r_{\mathrm{e} 2.1}$ and $r_{\mathrm{e} 3.7}$ suggesting the potential for either height (within cloud) dependent effects or the possibility that retrievals from this band are less reliable. The work required to solve these issues is also beyond the scope of this paper.

\subsection{The effect of cloud heterogeneity}

As discussed in Sect. 4.1, Figure 3c shows that the distributions of $\sigma_{\mathrm{CTT}}$ at low and high $\theta_{0}$ are very similar suggesting that the diurnal cycle is having little physical impact on this aspect of cloud heterogeneity. Therefore we might expect that for a given $\sigma_{\mathrm{CTT}}$, the subsets of clouds at low and high $\theta_{0}$ are likely to be physically similar, so that any differences in the retrieved $\tau$ and $r_{\mathrm{e}}$ are primarily due to retrieval artifacts.

We now examine the variation of $\tau, r_{\mathrm{e}}$ and $N_{\mathrm{d}}$ as a function of both $\sigma_{\text {CTT }}$ and $\gamma_{\tau}$. The restrictions 1-5 described in Sect. 3.3.2 still apply for these results.

\subsubsection{Cloud heterogeneity effects on optical depth}

Figure 11a shows mean $\tau$ as a function of $\sigma_{\mathrm{CTT}}$, at low VZA values of $<41.4^{\circ}$ for both low and high $\theta_{0}$. Figure $11 \mathrm{~b}$ shows the $\tau$ difference between high and low $\theta_{0}$ vs $\sigma_{\mathrm{CTT}}$. In the lower range of $\sigma_{\mathrm{CTT}}(<\sim 0.625-0.875 \mathrm{~K}) \tau$ increases as $\sigma_{\mathrm{CTT}}$ decreases for both low and high $\theta_{0}$. The increase is much larger for high $\theta_{0}\left(58 \%\right.$ increase between $\sigma_{\mathrm{CTT}}=$ 0.875 and $\sigma_{\mathrm{CTT}}=0.125 \mathrm{~K}$ ) than for low $\theta_{0}$ (an increase of $27 \%$ over the same range). At higher $\sigma_{\mathrm{CTT}}, \tau$ is approximately constant within the error range. It is evident that the increase in $\tau$ between low and high $\theta_{0}$ occurs at all values of $\sigma_{\text {CTT }}$. However, the increase is greatest at low values of $\sigma_{\mathrm{CTT}}$, i.e. when the cloud tops are more homogeneous.

These results are surprising as previous work (Loeb et al., 1997; Varnai and Davies, 1999) has suggested that a "bumpy" cloud top was the most likely explanation for the increase in $\tau$ with increasing $\theta_{0}$. If that were the case then it might be expected that $\tau$ would increase with increasing $\sigma_{\text {CTT }}$ at high $\theta_{0}$, that the $\tau$ increase with $\theta_{0}$ would be greater at higher $\sigma_{\mathrm{CTT}}$, and that at low $\sigma_{\mathrm{CTT}}$ there would be little difference in $\tau$ between low and high $\theta_{0}$ cases.

One possible explanation is that subpixel variability is causing $\tau$ decreases, as suggested by M06 and Z12, and so this may be counteracting the expected increase due to resolved scale heterogeneity. Another possible explanation is that the actual (i.e. as opposed to the retrieved) $\tau$ of the clouds was higher at lower $\sigma_{\mathrm{CTT}}$. Physically higher $\tau$ values at low $\sigma_{\text {CTT }}$ might be expected to lead to a greater $\tau$ bias between low and high $\theta_{0}$ (Loeb and Davies, 1996, 1997; Loeb and Coakley, 1998), as seen in Fig. 11. This seems likely to be a factor given that an increase of $\tau$ with decreasing $\sigma_{\mathrm{CTT}}$ was 
(a)

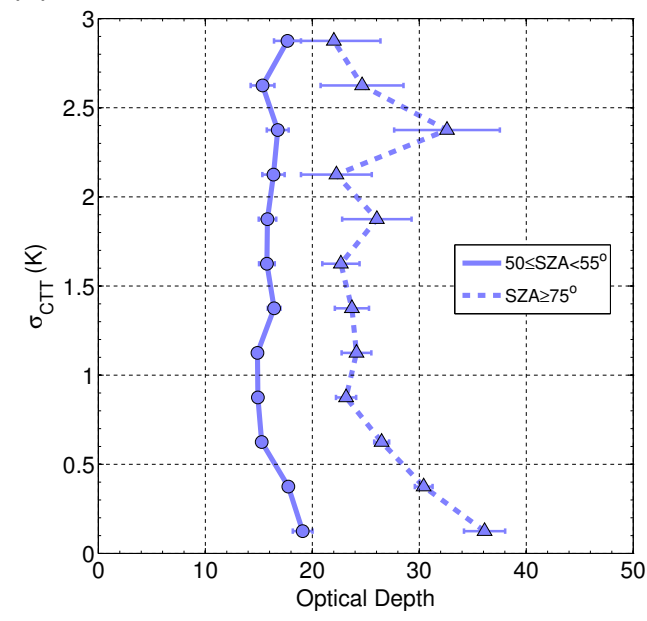

(b)

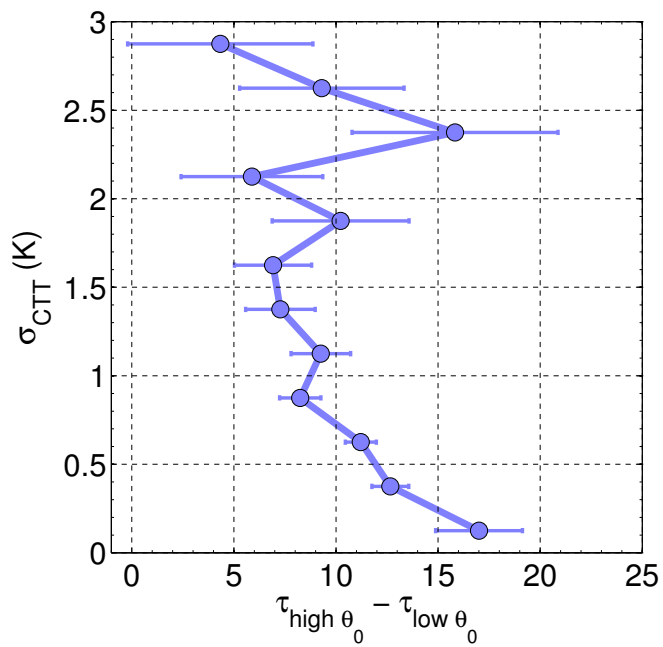

Figure 11. (a) Optical depth vs. $\sigma_{\mathrm{CTT}}$ for low VZA $(\leq 41.4)$ and for different solar zenith angles (SZA or $\theta_{0}$, see the legend). Other data restrictions are described in the text. (b) Differences in optical depth between high and low $\theta_{0}$.

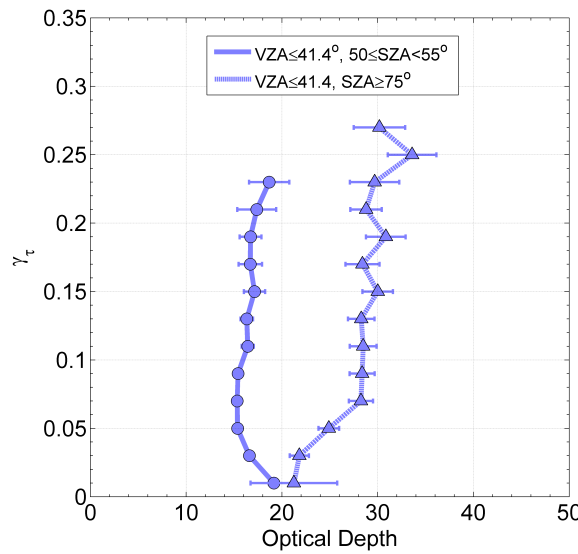

(a) Mean $\tau$

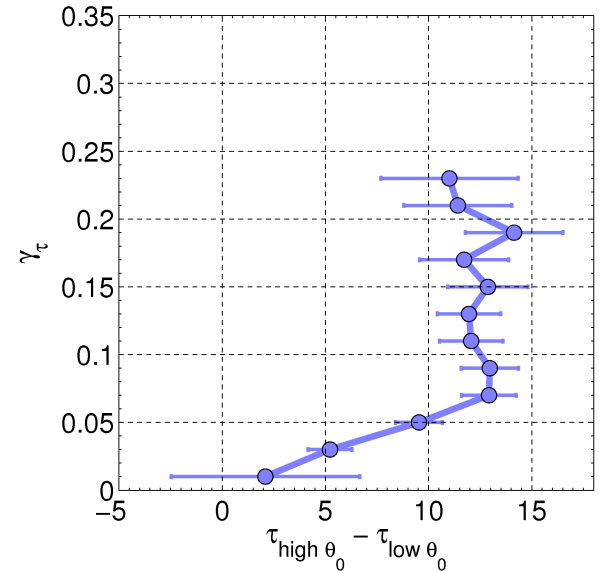

(b) High SZA minus low SZA.

Figure 12. As for Fig. 11 except for optical depth vs. $\gamma_{\tau}$, where $\gamma_{\tau}$ is a measure of cloud heterogeneity based on the variability of the retrieved $1 \mathrm{~km}$ cloud optical depth. Low values of $\gamma_{\tau}$ indicate more homogeneity.

observed at low $\theta_{0}$, where our results indicate that $\theta_{0}$ related biases should be small.

However, other factors are also likely at play and are now discussed through the examination of the effect of using $\gamma_{\tau}$ as a measure of cloud heterogeneity (see Sect. 3.2). This parameter has the advantage that it is calculated using $1 \mathrm{~km}$ resolution $\tau$ data and so can capture variability at smaller scales than $\sigma_{\mathrm{CTT}}$, which uses $5 \mathrm{~km}$ data. The disadvantage is that $\tau$ is a retrieved quantity and so $\gamma_{\tau}$ is subject to heterogeneity that is introduced through retrieval errors rather than representing solely physical cloud heterogeneity. CTT values are also retrieved and so may also suffer some heterogeneity bi- ases. However, these are likely to be significantly less than those for $\tau$ retrievals.

Figure 12 shows that at low $\theta_{0}, \tau$ varies with $\gamma_{\tau}$ in a similar way to how it varies with $\sigma_{\mathrm{CTT}}$. However, in contrast to when $\sigma_{\text {CTT }}$ was used as a measure of heterogeneity, there is little increase in $\tau$ between low and high $\theta_{0}$ for the lowest heterogeneity values. For high $\theta_{0}$ there is also a fairly monotonic increase in $\tau$ with $\gamma_{\tau}$ over the lower range of the $\gamma_{\tau}$ values sampled. This is interesting since for $\gamma_{\tau}, \theta_{0}$ biases therefore increase with heterogeneity, which would be the expected result if 3-D radiative effects played a role in causing the $\theta_{0}$ biases. 


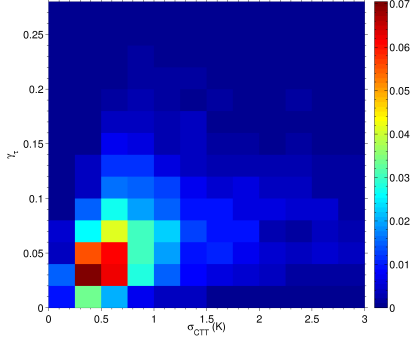

(a) $50 \leq \mathrm{SZA}<55^{\circ}$

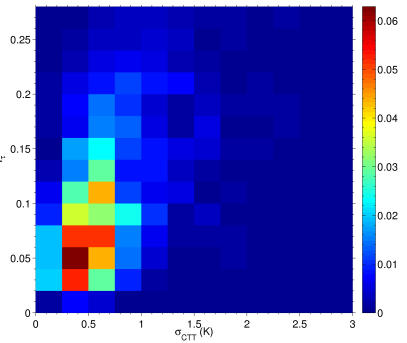

(b) $\mathrm{SZA} \geq 75^{\circ}$.
Figure 13. 2-D histogram of $\gamma_{\tau}$ vs. $\sigma_{\mathrm{CTT}}$ for low (a) and high (b) $\theta_{0}$ ranges.

We now examine the relationship between $\gamma_{\tau}$ and $\sigma_{\text {CTT }}$. Fig. 13 shows the 2-D histograms for these two parameters for both low and high $\theta_{0}$. It shows that at low $\theta_{0}$ (Fig. 13a) there is a lot of scatter with both low and high $\gamma_{\tau}$ values occurring for the intermediate $\sigma_{\mathrm{CTT}}$ range. The correlation coefficient in this case is only 0.29 . From the figure it appears that there are two branches in the scatter of the data; one for which $\gamma_{\tau}$ increases rapidly with increasing $\sigma_{\text {CTT }}$ and one for which there are only small increases in $\gamma_{\tau}$. We have examined this plot for smaller ranges of viewing angles and relative azimuth angles and found broadly the same result, indicating that the scatter is not caused by variation in viewing geometry. Thus the results are suggestive that, at low $\theta_{0}$, there is variability in the $1 \mathrm{~km}$ resolution radiative field (as captured by $\gamma_{\tau}$ ) that is not predicted well by the physical cloud top height variability from $5 \mathrm{~km}$ resolution data (as captured by $\sigma_{\text {CTT }}$ ).

Figure 13b shows the same result at high $\theta_{0}$. This broadly shows only a single relationship between $\sigma_{\mathrm{CTT}}$ and $\gamma_{\tau}$ with considerably larger values of $\gamma_{\tau}$ for a given $\sigma_{\text {CTT }}$ than at low $\theta_{0}$. Thus there is less scatter and a higher correlation coefficient of 0.45 . Figure 14 shows the mean $\gamma_{\tau}$ values for each bin of $\sigma_{\text {CTT }}$. The results are binned by $\sigma_{\text {CTT }}$ since it was shown in Fig. $3 c$ that this does not change much between low and high $\theta_{0}$. In general there is an increase in $\gamma_{\tau}$ with increasing $\sigma_{\mathrm{CTT}}$ at both low and high $\theta_{0}$. However, for a given $\sigma_{\mathrm{CTT}}$, $\gamma_{\tau}$ is larger at high $\theta_{0}$ showing that the increase in $\theta_{0}$ has induced an increase in radiative heterogeneity. The greater degree of correlation between $\sigma_{\text {CTT }}$ and $\gamma_{\tau}$ at high $\theta_{0}$ indicates that physical cloud top variability as diagnosed from $5 \mathrm{~km}$ data is more representative of $1 \mathrm{~km}$ resolution radiative variability than at low $\theta_{0}$.

However, considerable scatter still remains, suggesting that other factors, such as physical cloud top variability, at smaller scales than those captured using $5 \mathrm{~km}$ data are important. Extinction variations inside the cloud (without cloud top height variability) could also play a role, although this was found to have a small effect in Loeb et al. (1997) and Varnai and Davies (1999). Further work is needed to elucidate the relative merits of these explanations, which is beyond the scope of the observational data set used in this study.

\subsubsection{Cloud heterogeneity effects on effective radius}

Figure 15a and $\mathrm{b}$ show $r_{\mathrm{e}}$ for the different wavelengths vs. $\sigma_{\text {CTT }}$ at low and high $\theta_{0}$, respectively. Note that the results shown here for $r_{\mathrm{e}}$ are very similar whether $\sigma_{\mathrm{CTT}}$ or $\gamma_{\tau}$ is used as a measure of heterogeneity. The figure shows $r_{\mathrm{e}}$ values that decrease with increasing $\sigma_{\mathrm{CTT}}$ (i.e. increasing cloud top heterogeneity) for all wavelengths. However, $r_{\mathrm{e} 3.7}$ experiences the largest decrease and $r_{\mathrm{e} 1.6}$ experiences only small changes. At low $\sigma_{\mathrm{CTT}}, r_{\mathrm{e} 3.7}>r_{\mathrm{e} 2.1}>r_{\mathrm{e} 1.6}$, which is actually what would be expected given the increased penetration depth of the shorter-wavelength bands relative to the longer-wavelength ones and an assumed increase of droplet size with height (e.g. see Platnick, 2000). The contrast to the usual MODIS observation of $r_{\mathrm{e} 3.7}<r_{\mathrm{e} 2.1}<r_{\mathrm{e} 1.6}$ (e.g. Zhang and Plantnick, 2011) raises the possibility that the latter is caused by cloud top heterogeneity and that for homogeneous cloud tops (at low $\theta_{0}$ ) the $r_{\mathrm{e}}$ retrievals are more reliable and less prone to artifacts. Again, though, we have to bear in mind the possibility of physical cloud changes with $\sigma_{\text {CTT }}$.

The high $\theta_{0}$ results follow a similar pattern with a larger $r_{\mathrm{e}}$ decrease with increasing $\sigma_{\mathrm{CTT}}$ for $r_{\mathrm{e} 3.7}$ and $r_{\mathrm{e} 2.1}$ compared to $r_{\mathrm{e} 1.6}$. In fact, in the lower range of $\sigma_{\mathrm{CTT}}(<0.6 \mathrm{~K}) r_{\mathrm{e} 1.6}$ actually increases slightly with $\sigma_{\mathrm{CTT}}$. The convergence of $r_{\mathrm{e} 1.6}$, $r_{\mathrm{e} 2.1}$ and $r_{\mathrm{e} 3.7}$ at the lowest $\sigma_{\mathrm{CTT}}$ value is probably fortuitous and likely due to the trends with $\sigma_{\text {CTT }}$ of the different wavelength $r_{\mathrm{e}}$ values. Such convergence also occurs in Fig. 15a, although at a higher $\sigma_{\mathrm{CTT}}$ value. The difference can likely be put down to the effect of $\theta_{0}$ since Fig. $3 \mathrm{c}$ suggests that the low and high $\theta_{0}$ clouds would be physically similar at a given $\sigma_{\text {CTT }}$.

Additionally, the $r_{\mathrm{e}}$ values at high $\theta_{0}$ are generally lower than, or similar to, those at low $\theta_{0}$ for any given $\sigma_{\mathrm{CTT}}$, with the differences being considerably greater for $r_{\mathrm{e} 3.7}$ and $r_{\mathrm{e} 2.1}$ than for $r_{\mathrm{e} 1.6}$. The relative lack of change of $r_{\mathrm{e} 1.6}$ with $\theta_{0}$ and $\sigma_{\text {CTT }}$ again raises the possibility that this wavelength might be less susceptible to $r_{\mathrm{e}}$ artifacts caused by cloud top heterogeneity at high $\theta_{0}$. It also might be an argument against physical droplet size variations with $\sigma_{\text {CTT }}$. For the other wavelengths, the decreases in $r_{\mathrm{e}}$ between low and high $\sigma_{\mathrm{CTT}}$ are large, with the maximum decrease being $4.3 \mu \mathrm{m}(35 \%)$ in the case of $r_{\mathrm{e} 3.7}$ at high $\theta_{0}$. Given the sensitivity of $N_{\mathrm{d}}$ to $r_{\mathrm{e}}$ this is likely to have a large impact on the retrieved $N_{\mathrm{d}}$.

Earlier it was mentioned that the changes in $r_{\mathrm{e}}$ with heterogeneity were similar at both low and high $\theta_{0}$ whether measured by $\sigma_{\text {CTT }}$ or $\gamma_{\tau}$. This is likely to only be possible if the two parameters are correlated and if $r_{\mathrm{e}}$ changes with one parameter generally act in the same direction as with the other. Therefore it seems that $\gamma_{\tau}$ explains little extra variability in $r_{\mathrm{e}}$ compared to $\sigma_{\mathrm{CTT}}$. This in contrast to the situation with $\tau$ for high $\theta_{0}$ (but not for low $\theta_{0}$ ). 


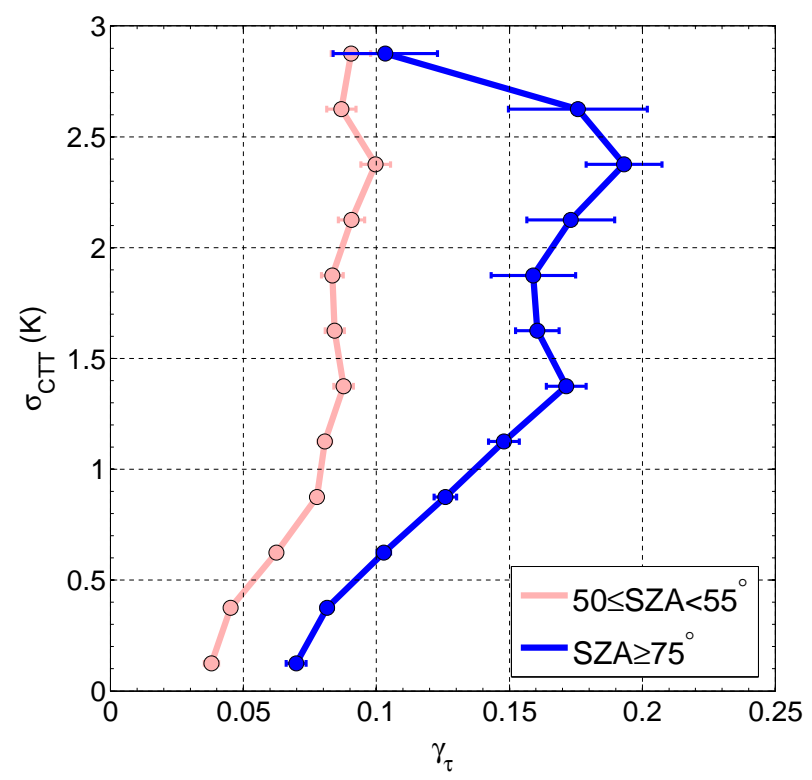

Figure 14. Mean $\gamma_{\tau}$ for each $\sigma_{\mathrm{CTT}}$ bin from Fig. 13 .

\subsubsection{Cloud heterogeneity effects on droplet concentration}

Similar plots to Fig. 15, but for $N_{\mathrm{d}}$, are shown in Fig. 16a and $\mathrm{b}$. Interestingly, in the low $\theta_{0}$ case, at low $\sigma_{\mathrm{CTT}}, N_{\mathrm{d}}$ values for all three wavelengths are very similar and there is little variation with $\sigma_{\mathrm{CTT}}$. There is an increase and divergence amongst the wavelengths at higher $\sigma_{\mathrm{CTT}}$, although the error bars also get larger. The increases from the lowest to highest $\sigma_{\mathrm{CTT}}$ value are 25,40 and $71 \%$ in the $r_{\mathrm{e} 1.6}, r_{\mathrm{e} 2.1} r_{\mathrm{e} 3.7}$ cases, respectively.

For the high $\theta_{0}$ case, $N_{\mathrm{d}}$ values are higher than for low $\theta_{0}$ for any given $\sigma_{\text {CTT }}$ value as expected from the $\tau$ and $r_{\mathrm{e}}$ results and from the results of Sect. 4.2.3. As for at low $\theta_{0}$, though, $N_{\mathrm{d}}$ is similar for the three wavelengths at low $\sigma_{\text {CTT }}$ and there is little variation of $N_{\mathrm{d}}$ with $\sigma_{\mathrm{CTT}}$. However, compared to at low $\theta_{0}, N_{\mathrm{d}}$ from the different wavelengths diverge at a lower $\sigma_{\text {CTT }}$ and at high $\sigma_{\text {CTT }}$ they diverge more widely and produce much higher $N_{\mathrm{d}}$ values - although, again, the error bars are large at high $\sigma_{\text {CTT }}$ due to a lack of samples. The increases in $N_{\mathrm{d}}$ between the lowest $\sigma_{\text {CTT }}$ value and $\sigma_{\text {CTT }}=2.6$, where the maximum $N_{\mathrm{d}}$ occurs, are $19,69,117 \%$ for the $r_{\mathrm{e} 1.6}$, $r_{\mathrm{e} 2.1} r_{\mathrm{e} 3.7}$ cases, respectively. Thus at both low and high $\theta_{0}$ the changes in $N_{\mathrm{d}}$ are smaller for $r_{\mathrm{e} 1.6}$.

It is interesting that at both low and high $\theta_{0}$ there is little change in $N_{\mathrm{d}}$ with $\sigma_{\text {CTT }}$ for low $\sigma_{\text {CTT }}$, as well as little difference between $N_{\mathrm{d}}$ from the different wavelengths. The constant $N_{\mathrm{d}}$ is due to the cancellation of an increasing $\tau$ and increasing $r_{\mathrm{e}}$ as $\sigma_{\mathrm{CTT}}$ decreases. Since we might expect retrievals to be less prone to retrieval artifacts at low $\sigma_{\mathrm{CTT}}$, the increase in $\tau$ with decreasing $\sigma_{\text {CTT }}$ might suggest that the more homogeneous clouds are actually physically thicker with a corresponding higher $\tau$ and higher $r_{\mathrm{e}}$, and thus that the $\tau$ and $r_{\mathrm{e}}$ changes are physical rather than due to retrieval artifacts. Also, it is feasible that $N_{\mathrm{d}}$ might be the same for homogeneous and heterogeneous clouds if the aerosol supply was similar for both cases, which would be consistent with the above result. However, heterogeneity is also known to be associated with increased precipitation and thus an increased CCN sink and might also be associated with altered updraft speeds, which would alter $N_{\mathrm{d}}$ activation. Shedding further light on this is difficult, however, without further observations of the clouds in question.

For low $\theta_{0}$, when using $\gamma_{\tau}$ as the heterogeneity parameter the results are similar to those using $\sigma_{\mathrm{CTT}}$, as would be expected from the similar variation of $\tau$ and $r_{\mathrm{e}}$ with both $\sigma_{\mathrm{CTT}}$ and $\gamma_{\tau}$. At high $\theta_{0}$ the lower $\tau$ values at low $\gamma_{\tau}$ (and high $\tau$ at high $\gamma_{\tau}$ ) cause $N_{\mathrm{d}}$ to increase monotonically with $\gamma_{\tau}$ (not shown).

\subsection{Attribution of $N_{\mathrm{d}}$ changes with $\theta_{0}$ to $\tau$ and $r_{\mathrm{e}}$ changes}

It would be useful to be able to determine whether the changes in $N_{\mathrm{d}}$ that occur with increasing $\theta_{0}$ were mainly due to changes in $\tau$ or changes in $r_{\mathrm{e}}$. As shown already, the means of both quantities change with increasing $\theta_{0}$ in the direction that causes an $N_{\mathrm{d}}$ increase, and so both are likely to contribute to some degree. Here we estimate the individual effects using a sensitivity analysis based upon Latin Hypercube Sampling for the change in $N_{\mathrm{d}}$ between low and high $\theta_{0}$. The details of this are described in Appendix D. Here we just discuss the main results, which are presented in Table 1. The main foci of the discussion here are the $\Delta N_{\Delta \text { re }}$ and $\Delta N_{\Delta \tau}$ values, which are the relative change in $N_{\mathrm{d}}$ between low and high $\theta_{0}$ due to, respectively, changes in $r_{\mathrm{e}}$ only and changes in $\tau$ only (see Eq. D1).

When considering the whole cloud population, the results show that for the $1.6 \mu \mathrm{m}$ band the contribution from changes in the $\tau$ distribution between low and high $\theta_{0}$ have an effect on $N_{\mathrm{d}}$ that is roughly 5-6 times larger than that from $r_{\mathrm{e}}$ changes. This is perhaps not a surprise given the relative lack of change in $r_{\mathrm{e}}$ with $\theta_{0}$ for that band. The $r_{\mathrm{e}}$ sensitivity is greater for the other bands; for the $2.1 \mu \mathrm{m}$ band $\Delta N_{\Delta \tau}$ is a factor of two larger than $\Delta N_{\Delta r_{\mathrm{e}}}$, whereas for the $3.7 \mu \mathrm{m}$ band it is only $40 \%$ larger. The greater sensitivity of $N_{\mathrm{d}}$ to $\tau$ biases between low and high $\theta_{0}$ may be initially unexpected given the fact that the power to which $r_{\mathrm{e}}$ is raised to in Eq. (A1) is five times greater than that for $\tau$.

For the high $\sigma_{\text {CTT }}$ cases (i.e. for the more heterogeneous clouds), however, the balance between $\Delta N_{\Delta \tau}$ and $\Delta N_{\Delta r_{\mathrm{e}}}$ shifts towards $\Delta N_{\Delta r_{\mathrm{e}}}$. At low VZA, $N_{\Delta \tau}$ is 2.5 times larger than $\Delta N_{\Delta r_{\mathrm{e}}}$ for the $1.6 \mu \mathrm{m}$ band. However, for the 2.1 and $3.7 \mu \mathrm{m}$ bands the sensitivity to $r_{\mathrm{e}}$ is greater than the $\tau$ sensitivity; $\Delta N_{\Delta r_{\mathrm{e}}}$ is $16 \%$ larger than $\Delta N_{\Delta \tau}$ for $2.1 \mu \mathrm{m}$ and $54 \%$ larger for $3.7 \mu \mathrm{m}$. 
(a)

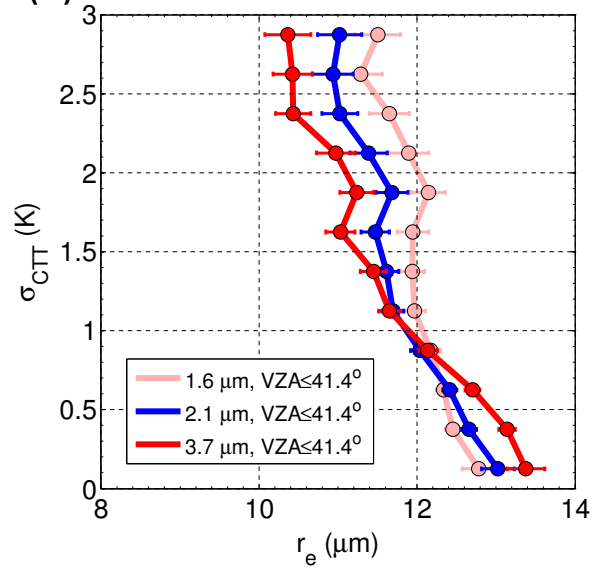

(b)

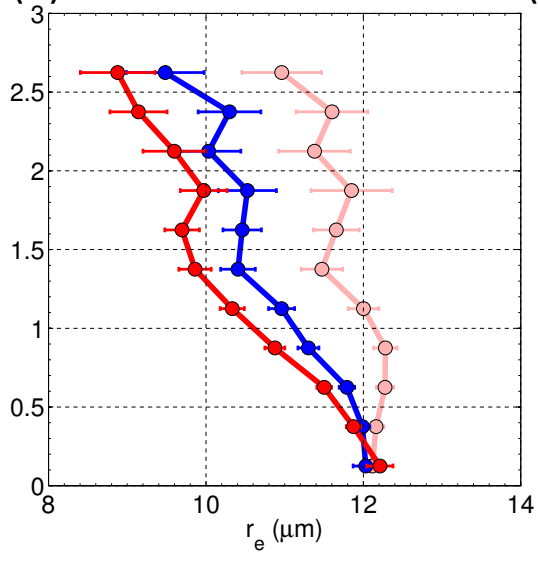

(c)

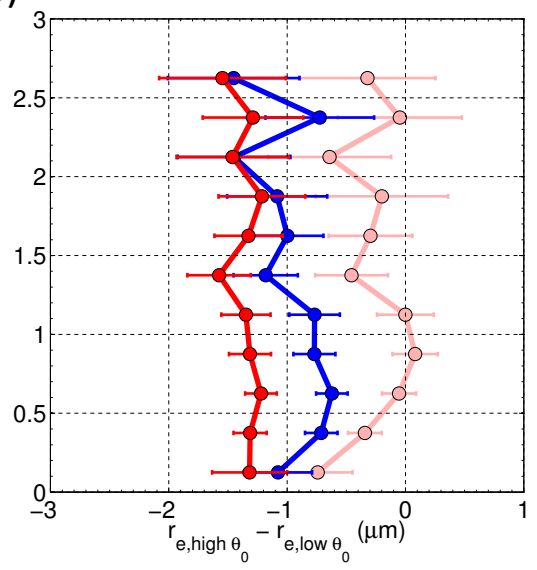

Figure 15. As for Fig. 11 except for effective radius for low $\theta_{0}(\mathbf{a})$, high $\theta_{0}(\mathbf{b})$, and the difference between $r_{\mathrm{e}}$ at high and low $\theta_{0}(\mathbf{c})$.

(a)

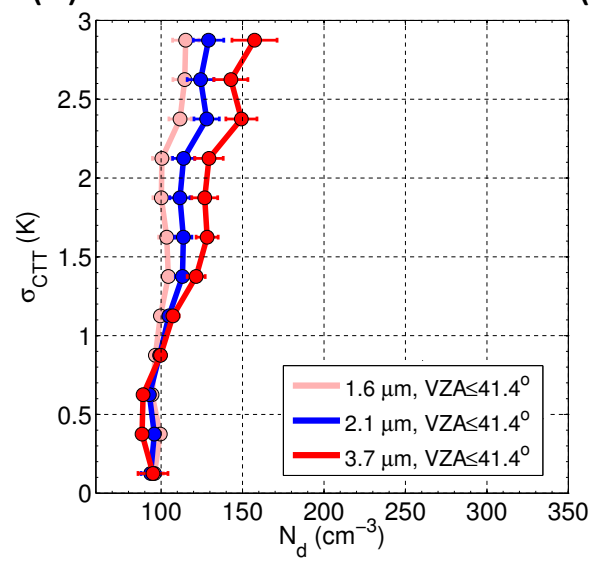

(b)

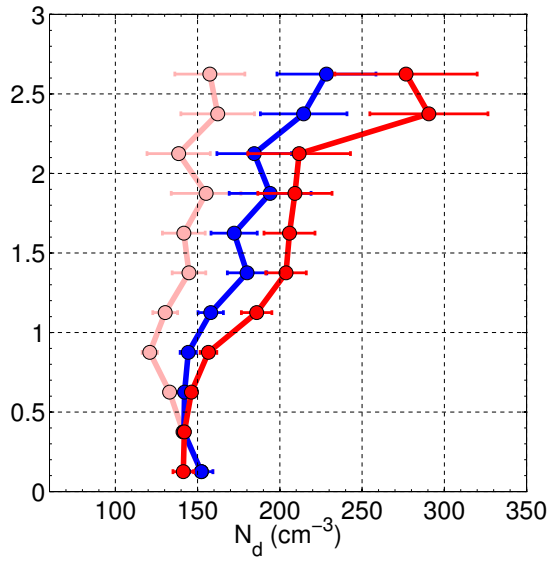

(c)

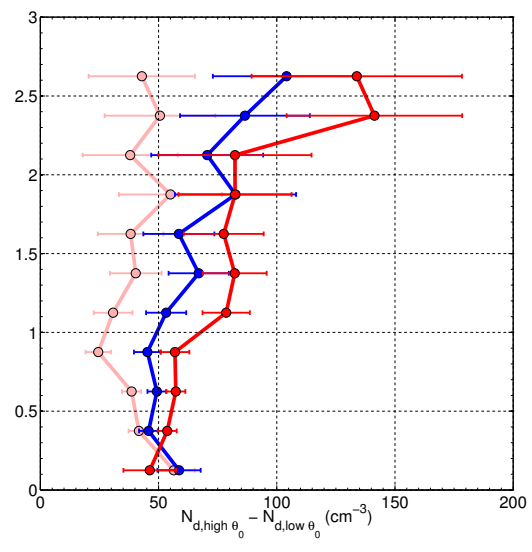

Figure 16. As for Fig. 15 except for droplet number concentration.

Overall, these results suggest that biases in $\tau$ and $r_{\mathrm{e}}$ between low and high $\theta_{0}$ can both be important causes of the increase in $N_{\mathrm{d}}$ at high $\theta_{0}$, depending upon the $r_{\mathrm{e}}$ band and the cloud heterogeneity.

\section{Discussion}

In this section we focus on a discussion of potential explanations of the observed changes of $r_{\mathrm{e}}$ with $\theta_{0}$ and cloud heterogeneity. We do not discuss $\tau$ effects because there has been much discussion on the causes of $\tau$ biases at high $\theta_{0}$ in the literature (see Sect. 2.1), whereas there has been much less work focused upon $r_{\mathrm{e}}$ effects.

\subsection{Potential explanations for the $r_{\mathrm{e}}$ decrease with $\theta_{0}$}

Here we discuss possible reasons for the decrease in $r_{\mathrm{e}}$ that we observe as $\theta_{0}$ increases. Table 3 summarizes three poten- tial effects that could be a cause of $r_{\mathrm{e}}$ changes with $\theta_{0}$ and the direction of their effects on $r_{\mathrm{e}}$. These mechanisms are discussed in more detail below. It should be noted that there may be additional effects in operation that are not listed here. The three mechanisms are as follows:

1. The averaging scale $r_{\mathrm{e}}$ bias. As discussed in Sect. 2.2, M06 and Z12 found opposite signs for the effect of subpixel averaging on $r_{\mathrm{e}}$ retrievals and it was suggested in Sect. 2.2 that a potential cause of the disagreement may be that the radiative transfer was performed at a higher $\theta_{0}$ in M06 than in Z12. This indicates that varying $\theta_{0}$ may influence the sign of $r_{\mathrm{e}}$ changes during subpixel averaging. In order to cause a negative $r_{\mathrm{e}}$ bias relative to the true $r_{\mathrm{e}}$ it would be required that there was a high degree of $R_{\mathrm{ab}}$ variability within the scale of the pixel. Z12 showed that clouds physically tend to have more $\tau$ variability than $r_{\mathrm{e}}$ variability over the scale of a MODIS pixel and so an overall negative bias from this effect due 

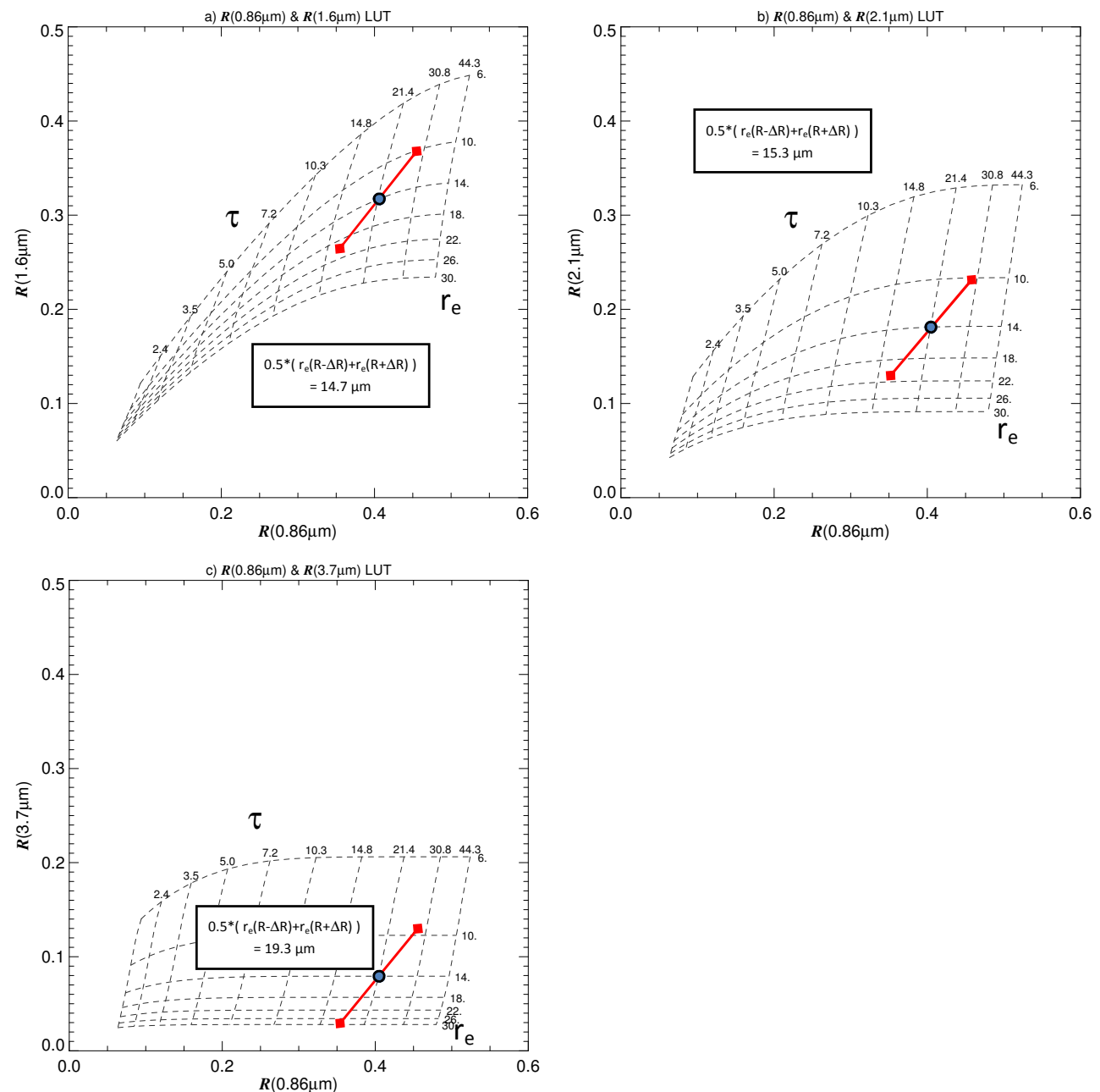

Figure 17. An example of the effect of 3-D effects on MODIS retrievals at small averaging scales. 3-D effects at high $\theta_{0}$ are assumed to cause an equal increase and decrease $(\Delta R)$ in the reflectances of both the absorbing band $\left(R_{\mathrm{ab}}\right)$ and the non-absorbing band $\left(R_{\mathrm{nab}}\right)$. For this demonstration it is assumed that there are an equal number of small-scale cloud elements all with $r_{\mathrm{e}}=14 \mu \mathrm{m}$ and $\tau=23$.6. Retrievals are then made on the reflectances that have been distorted by the 3-D effects using MODIS lookup tables (LUTs) that are used for converting non-absorbing $(0.86 \mu \mathrm{m})$ and absorbing reflectance pairs into $\tau$ and $r_{\mathrm{e}}$. These are shown for $\theta_{0}=79^{\circ}$ and a nadir viewing angle: (a) is for the $1.6 \mu \mathrm{m}$ absorbing band, (b) for $2.1 \mu \mathrm{m}$ and (c) for $3.7 \mu \mathrm{m}$. It can be seen that in all cases the retrieved $r_{\mathrm{e}}$ would be greater than the true $r_{\mathrm{e}}$ of $14 \mu \mathrm{m}$. See Sect. 5.1 for further details.

to physical heterogeneity seems unlikely. Rather, it is likely that in M06 there was a high degree of $R_{\mathrm{ab}}$ variability caused by $3-\mathrm{D}$ radiative effects at high $\theta_{0}$ due to the increased interception of photons by cloud sides and extra illumination and shadowing effects when the Sun is low in the sky (e.g. see Loeb et al., 1997). As explained in M06, this would have the effect of causing an overestimate of $r_{\mathrm{e}}$ at small averaging scales, with the positive bias reducing towards zero as the averaging scale is increased.

For the lower $\theta_{0}$ results of Z12, increased averaging scales led to an increasingly positive $r_{\mathrm{e}}$ change. Therefore at sufficiently large averaging scales it is likely possible for $r_{\mathrm{e}}$ values at high $\theta_{0}$ to be lower than those at low $\theta_{0}$, as observed in our study. However, the larger reduction in $r_{\mathrm{e} 3.7}$ relative to $r_{\mathrm{e} 2.1}$ as observed in our study gives some indications that this averaging scale effect is unlikely to be the dominant cause of the $r_{\mathrm{e}}$ change that we observed. This is discussed in the next section (Sect. 5.2).

2. The plane parallel (PP) $r_{\mathrm{e}}$ bias. As described in Sect. 2.1, modelled non-absorbing reflectances $\left(R_{\mathrm{nab}}\right)$ from realistic heterogeneous clouds using 3-D radiative transfer and those produced from PP clouds (of the same optical depth) are found to change in the opposite directions as $\theta_{0}$ increases. This leads to an increasingly positive $\tau$ bias with increasing $\theta_{0}$ when using the PP model to make retrievals. If differences in absorbing 
wavelength reflectances $\left(R_{\mathrm{ab}}\right)$ between heterogeneous and PP clouds varied in a similar manner with $\theta_{0}$ then this would lead to a negative $r_{\mathrm{e}}$ bias (because $r_{\mathrm{e}}$ reduces with increasing $R_{\mathrm{ab}}$ ) at high $\theta_{0}$ and might provide another potential explanation for the observed result. Indeed Loeb and Coakley (1998) provide some evidence that $R_{\mathrm{ab}}$ may respond to 3-D radiative effects in a similar manner to $R_{\text {nab }}$.

3. The droplet size distribution (DSD) bias. Zhang (2013) found that wider DSDs than those assumed by the MODIS retrieval (MODIS assumes a single DSD width) would lead to a negative bias in the retrieved $r_{\mathrm{e}}$. We can speculate that this effect may be more pronounced at higher $\theta_{0}$, although further work is needed to investigate this.

In reality it is likely that combinations of all of these effects will occur to cause increases or decreases in $r_{\mathrm{e}}$ depending upon circumstances. Further work is needed to elucidate the signs and magnitudes of these effects under different viewing geometries, cloud fields, etc.

\subsection{Potential explanations for why the $r_{\mathrm{e}}$ reduction with $\theta_{0}$ varies amongst MODIS bands}

Another interesting aspect of the current work is the stronger observed decrease of $r_{\mathrm{e} 3.7}$ with increasing $\theta_{0}$ compared to $r_{\mathrm{e} 2.1}$, along with the lack of change of $r_{\mathrm{e} 1.6}$. Here we discuss possible reasons for this by considering the likely relative magnitudes of the three effects mentioned in the previous section for the different MODIS bands (see Table 3). We attempt to estimate these relative changes for effect 1 , although these estimates are fairly uncertain. For effects 2 and 3 there has been little previous work on this and such work is beyond the scope of this study and so we leave this as an unknown. Also, most previous work has only considered differences between $r_{\mathrm{e} 2.1}$ and $r_{\mathrm{e} 3.7}$.

As mentioned in the previous section, it seems likely that in the model results of M06, 3-D effects at high $\theta_{0}$ caused a positive bias in $r_{\mathrm{e}}$ when the averaging scale was small (effect 1 above and in Table 3). Interestingly, such an overestimate is likely to be larger for $r_{\mathrm{e} 3.7}$ than for $r_{\mathrm{e} 2.1}$. Figure 17 shows an example of why this is so. The assumption is made that there are a number of small regions of cloud with the same $r_{\mathrm{e}}(=14 \mu \mathrm{m})$ and $\tau(=21.4)$. These correspond to $R_{\mathrm{ab}}$ and $R_{\mathrm{nab}}$ values that can be determined using PP LUTs similar to those used operationally for MODIS retrievals. It is then assumed that 3-D radiative transfer at high $\theta_{0}$ causes the absorbing and non-absorbing reflectances of half of these regions to be decreased and half to be increased by the same amount, $\Delta R=0.05$, from the PP values. PP retrievals are then performed upon these distorted reflectances and it is found that the retrieved $r_{\mathrm{e}}$ increases relative to the true $r_{\mathrm{e}}$. However, the retrieved $r_{\mathrm{e} 3.7}$ is $4 \mu \mathrm{m}$ larger than $r_{\mathrm{e} 2.1}\left(r_{\mathrm{e} 2.1}=15.3 \mu \mathrm{m}, r_{\mathrm{e} 3.7}=19.3 \mu \mathrm{m}\right) . r_{\mathrm{e} 1.6}=14.7 \mu \mathrm{m}$ and so experiences the least bias.

Table 4 shows the magnitude of this effect at different VZA and $\phi$ and reveals that the bias from the true values and the difference between $r_{\mathrm{e} 3.7}$ and $r_{\mathrm{e} 2.1}$ is likely to be lower at high VZA $\left(50^{\circ}\right)$ than at nadir. The biases when $\phi=30^{\circ}$ are especially low, suggesting that 3-D effects are highly sensitive to the viewing geometry. The sign of the relative differences between the different bands is maintained at all the viewing geometries, with $r_{\mathrm{e} 3.7}>r_{\mathrm{e} 2.1}>r_{\mathrm{e} 1.6}$.

Thus for high-resolution retrievals at high $\theta_{0}, 3-\mathrm{D}$ effects are likely to cause on overestimate in $r_{\mathrm{e}}$. However, upon averaging reflectances over ever larger averaging scales the retrieved $r_{\mathrm{e}}$ would be expected to decrease towards the true value as the positive and negative reflectance changes start to cancel out. The above example suggests that for high $\theta_{0}$, at any given averaging scale we would expect $r_{\mathrm{e} 3.7}$ to be larger than $r_{\mathrm{e} 2.1}$. However, this is the opposite to what was what was found from the results presented earlier in this paper, which would indicate that 3-D effects of this type are not the sole cause of the observed changes in $r_{\mathrm{e}}$ as a function of $\theta_{0}$ and heterogeneity (see next section for further discussion on heterogeneity issues).

Some caveats here are that for real-world 3-D effects it may not be the case that $\Delta R$ values are the same for all of the non-absorbing bands and they may also be different for the absorbing and non-absorbing bands. $R_{\text {nab }}$ values for the $\tau$ and $r_{\mathrm{e}}$ values used for the PP LUTs tend to span a wider range of reflectance than $R_{\mathrm{ab}}$ values (e.g. see Fig. 17) and $R_{\mathrm{ab}}$ spans a wider range for the $2.1 \mu \mathrm{m}$ band compared to the $3.7 \mu \mathrm{m}$ band. Thus some $\Delta R$ differences may be expected from this. However, little has been reported on the relative magnitudes of $\Delta R$ as a function of wavelength and so it is difficult to assess the likely effects. Another caveat is that it may not be the case that positive $\Delta R$ values are the same as negative ones since that would mean that the overall reflectance change was zero on average, which according to the works cited in Sect. 2.1 (regarding the PP $r_{\mathrm{e}}$ bias) is not likely to be the case. In addition, the other effects mentioned (the PP $r_{\mathrm{e}}$ bias and the DSD bias) also have the potential to interact with these effects to produce the result observed in this paper. For example, Zhang (2013) showed that the decrease in $r_{\mathrm{e}}$ due to the effects of a wide DSD are likely to be greater for $r_{\mathrm{e} 3.7}$ than for $r_{\mathrm{e} 2.1}$, which is consistent with the results presented here for high $\theta_{0}$. However, it would be expected that this effect would also occur at low $\theta_{0}$ and so it is unclear whether this could lead to a change with $\theta_{0}$. Further work is required to investigate these matters, which is beyond the scope of this study. 


\subsection{Discussion on the observed changes in the retrieval of $r_{\mathrm{e}}$ with cloud heterogeneity}

The results described in Sect. 4.4.2 and discussed in Sect. 5.2 showed that a general decrease in $r_{\mathrm{e} 2.1}$ and $r_{\mathrm{e} 3.7}$ occurs with increasing cloud heterogeneity (whether measured using $\sigma_{\text {CTT }}$ or $\left.\gamma_{\tau}\right)$ for all the $\theta_{0}$ values studied here $\left(\theta_{0}>50^{\circ}\right)$, but that there was little change in $r_{\mathrm{e} 1.6} \cdot r_{\mathrm{e} 3.7}$ was also found to decrease at a faster rate than $r_{\mathrm{e} 2.1}$. Both $\theta_{0}$ increases and physical cloud top heterogeneity (as described by $\sigma_{\mathrm{CTT}}$ ) were found to cause increases in reflectance heterogeneity (as indicated from $\gamma_{\tau}$ ). Thus the observed greater decrease in $r_{\mathrm{e} 3.7}$ compared to $r_{\mathrm{e} 2.1}$ as $\sigma_{\mathrm{CTT}}$ increases and the results from Sect. 4.2.2 whereby $r_{\mathrm{e} 3.7}$ was the wavelength most strongly affected by $\theta_{0}$ changes, may both be related via cloud radiative heterogeneity. Also, the results suggest that $r_{\mathrm{e} 3.7}$ elicits a stronger response to heterogeneity than the other bands. However, a significant caveat is that subpixel heterogeneity is also likely to be important and it is not clear whether $\sigma_{\text {CTT }}$ or $\gamma_{\tau}$ are good indicators of subpixel heterogeneity due to them being calculated using $5 \mathrm{~km}$ and $1 \mathrm{~km}$ data, respectively. Also, these measures may not be good indicators of $R_{\mathrm{ab}}$ variability, which may be more important when considering $r_{\mathrm{e} 3.7}$ effects.

Another complicating factor here is that there may be some physical cloud changes that occur as a function of heterogeneity as was also indicated by the variation of $\tau$ with $\sigma_{\text {CTT }}$ and $\gamma_{\tau}$ at low $\theta_{0}$. This could alter the vertical profile of droplet radii and thus the relative $r_{\mathrm{e}}$ values from the different bands. At low $\theta_{0}$ the clouds with more homogeneous cloud tops had $r_{\mathrm{e} 3.7}>r_{\mathrm{e} 2.1}>r_{\mathrm{e} 1.6}$, which is actually what might be expected from a cloud in which $r_{\mathrm{e}}$ increased with height due to the increased penetration depth of the smaller wavelengths of light (e.g. see Platnick, 2000). Many studies have suggested that the differences between MODIS $r_{\mathrm{e} 1.6}$, $r_{\mathrm{e} 2.1}$ and $r_{\mathrm{e} 3.7}$ can impart information on the vertical structure of $r_{\mathrm{e}}$ near cloud top (Chang and Li, 2002, 2003; Chen et al., 2007; Seethala and Horvath, 2010; Nakajima et al., 2010a, b). It would be expected that $r_{\mathrm{e}}$ would increase monotonically with height in an idealized cloud with no entrainment occurring and no drizzle drops present. In reality both may occur and so may have the potential to reverse this gradient. The observation from Fig. 15a that the $r_{\mathrm{e} 1.6}, r_{\mathrm{e} 2.1}$ and $r_{\mathrm{e} 3.7}$ values are consistent with such a gradient reversal between low and high cloud heterogeneity values is interesting since more heterogeneous clouds are likely to be associated with more prevalent drizzle.

However, the work of Z12 and Zinner et al. (2010) suggests that precipitation is unlikely to have a large impact on $r_{\mathrm{e}}$ retrievals. In addition, theoretical work presented in King and Vaughan (2012) indicates that measurement and plane parallel modelling uncertainties are likely to be too large to accurately discern differences in the vertical variation of $r_{\mathrm{e}}$ using the MODIS bands available.
Despite the uncertainties in determining the relative importance of physical and retrieval artifacts as a function of heterogeneity in our results, it can be said that $\theta_{0}$ affects the relative values of $r_{\mathrm{e} 1.6}, r_{\mathrm{e} 2.1}$ and $r_{\mathrm{e} 3.7}$ at all values of heterogeneity, and therefore that $\theta_{0}$ effects will need to be considered if attempting to determine vertical variation information from MODIS observations.

\section{Summary and implications}

In this paper we have examined the effect of solar zenith angle $\left(\theta_{0}\right)$ on MODIS retrievals of $\tau, r_{\mathrm{e}}$ and $N_{\mathrm{d}}$, where the latter is a function of the two former quantities (Eq. A1). To do this we examined Arctic stratocumulus clouds in a region of the Norwegian/Barents Sea ( 72 to $75^{\circ} \mathrm{N},-3$ to $\left.48^{\circ} \mathrm{E}\right)$. This region has the advantage of being completely free of sea ice throughout the year, but yet it is far enough north to experience several Terra and Aqua overpasses per day. This means that $\theta_{0}$ retrieval effects can be examined in actual MODIS data by utilizing the diurnal cycle. Potential latitudinal and seasonal variations of cloud properties can be avoided by focusing upon a short time period (13-30 June) and upon a small latitude range. However, there is the possibility that there are physical changes of the clouds during the diurnal cycle. We argue that these changes are likely to be small because the diurnal cycle here is one of the weakest on Earth in terms of LWP variation (O'Dell et al., 2008), probably because the Sun is only below the horizon for a short period in this mid-summer period. We have also shown that the variation within our region of retrieved $N_{\mathrm{d}}$ with local time is more characteristic of a $\theta_{0}$ retrieval artifact than of a diurnal cycle.

In addition to this, we have looked for differences between low and high $\theta_{0}$ data points in quantities that give some information on the physical states of the clouds, but that are not affected by the types of optical retrieval bias that we are searching for. These include the MODIS cloud top temperature (CTT) and the variability of MODIS CTT $\left(\sigma_{\text {CTT }}\right)$. CTT and $\sigma_{\text {CTT }}$ PDFs are virtually identical for the low and high $\theta_{0}$ ranges, suggesting that there is little physical difference in the cloud populations at these different times of day in terms of cloud thickness and heterogeneity.

The results of the $\theta_{0}$ analysis showed that the mean $\tau$ was fairly constant between $\theta_{0}=50^{\circ}$ and $\sim 65-70^{\circ}$, but then increased rapidly with an increase of over $70 \%$ between the lowest and highest $\theta_{0}$. In contrast the change between the low and high viewing zenith angles (VZA) ranges was small at both low and high $\theta_{0}$. The change due to $\theta_{0}$ is consistent with previous studies on the effect of $\theta_{0}$ on $\tau$ (Loeb and Davies, 1996, 1997; Loeb and Coakley, 1998; Loeb et al., 1998, 1997; Varnai and Davies, 1999). From these studies it was ascertained that the bias arose through differences in how the reflectance of real (heterogeneous) clouds changed with $\theta_{0}$ relative to the plane parallel clouds used to model the reflectance $-\tau$ relationships used for retrievals. These studies 
suggested that the difference was mainly a result of variability in cloud top height rather than extinction variability. The lack of VZA sensitivity is perhaps more surprising, although studies have shown that the magnitude and sign of the effect of changing VZA is dependent upon whether back or forward scatter viewing angles are being employed and on the cloud thickness (Loeb and Davies, 1997; Loeb and Coakley, 1998; Loeb et al., 1998; Liang and Girolamo, 2013). The relative azimuth angles $(\phi)$ of the retrievals in this study were all side scatter viewing angles.

Larger increases in $\tau$ with $\theta_{0}$ were observed at lower cloud top heterogeneities, as measured by $\sigma_{\mathrm{CTT}}$, which was a little puzzling since the work mentioned above suggested that cloud top heterogeneity played a large role in causing $\theta_{0}$ biases. It seems likely that the clouds were physically thicker at low $\sigma_{\text {CTT }}$, which may have had some effect on the $\theta_{0}$ bias results. However, it is also true that this measure of cloud top heterogeneity is only capable of quantifying heterogeneity at scales resolved by the $5 \mathrm{~km}$ data resolution, which may not be the relevant scale for the effects in question. When using the variability of $1 \mathrm{~km} \tau$ data $\left(\gamma_{\tau}\right)$ as a heterogeneity parameter we obtained the expected result of increasing differences in $\tau$ between high and low $\theta_{0}$ as heterogeneity increased, suggesting that $\gamma_{\tau}$ is a better predictor of $\theta_{0}$ biases in $\tau$ than $\sigma_{\text {CTT }}$.

$r_{\mathrm{e}}$ values retrieved using the 2.1 and $3.7 \mu \mathrm{m}$ bands $\left(r_{\mathrm{e} 2.1}\right.$ and $r_{\mathrm{e} 3.7}$, respectively) were found to decrease with $\theta_{0}$, with effects starting at around $\theta_{0}=65-70^{\circ}$, which is consistent with the $\theta_{0}$ at which the $\tau$ increases occurred. At low $\theta_{0}$ the $r_{\mathrm{e}}$ values from the three different MODIS bands agree to within around $0.2 \mu \mathrm{m}$, whereas at high $\theta_{0}$ the spread is closer to $1 \mu \mathrm{m}$. The percentage changes of $r_{\mathrm{e}}$ with $\theta_{0}$ were considerably lower than those for $\tau$, being around $5 \%$ and $7 \%$ for $r_{\mathrm{e} 2.1}$ and $r_{\mathrm{e} 3.7}$, respectively. However, for $r_{\mathrm{e} 1.6}$ there was very little change with $\theta_{0}$. Larger decreases in $r_{\mathrm{e}}$, which depended upon the MODIS $r_{\mathrm{e}}$ band, were observed as the cloud top heterogeneity changed from low to high values; decreases of $25-30 \%$ for $r_{\mathrm{e} 3.7}, \sim 20 \%$ for $r_{\mathrm{e} 2.1}$ and $10 \%$ for $r_{\mathrm{e} 1.6}$. However, it is possible that the clouds were changing physically with cloud top heterogeneity and that such changes may affect the retrieved $r_{\mathrm{e}}$ as well. The use of $\gamma_{\tau}$ as a heterogeneity parameter did not change the results for $r_{\mathrm{e}}$.

Whilst the $r_{\mathrm{e}}$ changes are quite small they are not insignificant for the calculation of $N_{\mathrm{d}}$, since the equation relating $N_{\mathrm{d}}$ to $\tau$ and $r_{\mathrm{e}}$ implies a sensitivity to $r_{\mathrm{e}}$ changes that is five times greater than the sensitivity to $\tau$ changes. Using Latin hypercube sampling (LHS) sensitivity analysis we assessed the relative contributions of the $\tau$ increase and $r_{\mathrm{e}}$ decrease to the $N_{\mathrm{d}}$ changes between low and high $\theta_{0}$. The overall $N_{\mathrm{d}}$ increase between low and high $\theta_{0}$ varied between $\sim 40$ and $70 \%$ depending on MODIS band and VZA. When considering the studied cloud population as a whole, it was found that the $N_{\mathrm{d}}$ contribution from the $\tau$ biases and $r_{\mathrm{e}}$ biases were roughly comparable for $r_{\mathrm{e} 3.7}$. However, for the other $r_{\mathrm{e}}$ bands the $\tau$ changes were considerably more important (roughly twice the contribution for $r_{\mathrm{e} 2.1}$ and six times for $\left.r_{\mathrm{e} 1.6}\right)$. However, when considering only the more heterogeneous clouds, the importance of the $r_{\mathrm{e}}$ biases was considerably enhanced for both $r_{\mathrm{e} 2.1}$ and $r_{\mathrm{e} 3.7} ; \tau$ and $r_{\mathrm{e}}$ bias contributions were comparable for $r_{\mathrm{e} 2.1}$ and for $r_{\mathrm{e} 3.7}$ bias contributions from $r_{\mathrm{e}}$ were $\sim 50 \%$ greater.

\subsection{Implications for Level-3 retrievals and a new data set}

The analysis presented in this paper suggests that when $\theta_{0}$ is larger than around $65-70^{\circ}$, MODIS retrievals of $\tau, r_{\mathrm{e}}$ and $N_{\mathrm{d}}$ become unreliable due to optical artifacts, which suggests that such retrievals should not be used. This would unfortunately mean that large regions of the globe at higher latitudes would need to be excluded in their winter seasons when the Sun is low in the sky, unless it becomes possible to confidently tie biases to observable cloud properties (e.g. cloud variability, etc.), which might then allow some high $\theta_{0}$ data to be reliably used. The problem is relevant for the MODIS daily L3 product since this produces averages of $\tau$ and $r_{\mathrm{e}}$ over all overpasses that occur on a given day for which $\theta_{0}<81.4^{\circ}$. Some locations will experience several overpasses per day and thus retrievals will be made at a range of $\theta_{0}$ values. At some locations on a given day some of the daily overpasses will occur at $\theta_{0}$ near $65-70^{\circ}$ and therefore might not be affected by the biases seen here too greatly, but other overpasses will occur at much greater $\theta_{0}$. For these locations some "good" data are available, but for the L3 product they will be averaged in with "bad" data. Thus, taking the conservative approach it would be prudent to discard the daily averaged L3 value. This problem is more likely to occur as the number of daily overpasses increases, which is generally the case moving poleward. Analysis suggests that the most strongly affected regions/times for which both good and bad data will be contained in L3 will be those poleward of $\sim \pm 64^{\circ}$ for the spring and summer seasons. At higher latitudes and in the winter season there will still be L3 data for which $\theta_{0}>65-70^{\circ}$, but in those cases there will be no good data that is also salvageable. Overpasses with $\theta_{0}>65-70^{\circ}$ can occur at latitudes as low as $\sim 28^{\circ}$ in mid-winter and thus the $\theta_{0}$ bias problem has the potential to affect very large regions of the globe. Given this, an operational solution to the problem would ideally be sought in order to avoid the inclusion of high $\theta_{0}$ retrievals within daily L3 averages.

We have compiled our own version of the $\mathrm{L} 3$ product using similar procedures to those used for the operational product, but excluding data from overpasses $>65^{\circ}$. In a follow-on paper we will examine this data set in order to identify the main problem regions/times and we will also explore science problems relating to $N_{\mathrm{d}}$, but in the light of the $\theta_{0}$ biases identified here. 
We note that MODIS Collection 6 data sets are now being released (the data used in this study came from Collection 5.1). One significant difference is that quality assurance flags are no longer assigned, but rather new pixel-level uncertainty calculations are included that are intended to replace them. It is unclear whether this will account for $\theta_{0}$ effects, although generally MODIS uncertainty calculations have only accounted for instrumental measurement error rather than forward modelling error and so this is perhaps unlikely. It seems unlikely that the new uncertainty calculations will lead to the results presented here changing significantly since only pixels with the highest confidence quality assurance flags were used to calculate cloud properties. There will also be a $1 \mathrm{~km}$ resolution physical cloud height product that may be useful for assessing cloud top height variation at a higher resolution than that afforded by the $5 \mathrm{~km}$ resolution cloud top temperature product of Collection 5.1, if the technique used proves to be sufficiently accurate for low-level stratocumulus clouds.
Finally we should mention that whilst the analysis here has focused upon data from the MODIS instruments it is also likely that some of the $\theta_{0}$ effects described here would apply to $\tau$ and $r_{\mathrm{e}}$ retrievals from satellite instruments that use visible light at similar wavelengths along with forward retrieval models that assume PP clouds, such as the GOES imagers, SEVIRI, etc. However, the nature of the bias could potentially be altered somewhat by a variety of factors such as the use of different LUTs and the different instrument resolutions. The latter is likely important since it will alter the scale over which reflectances are averaged by the instrument. 


\section{Appendix A: The method used to estimate droplet concentration}

The formula for the estimation of $N_{\mathrm{d}}$ from $\tau$ and $r_{\mathrm{e}}$ as derived in Boers et al. (2006) and B07 is

$N_{\mathrm{d}}=\frac{2 \sqrt{10}}{k \pi Q^{3}}\left(\frac{c(T, P) \tau}{\rho_{\mathrm{w}} r_{\mathrm{e}}^{5}}\right)^{1 / 2}$

$k=\left(r_{\mathrm{v}} / r_{\mathrm{e}}\right)^{3}$,

where $\tau$ is the cloud optical thickness, $r_{\mathrm{e}}$ and $r_{\mathrm{v}}$ are the cloud top effective and volume mean radius, respectively, $k$ is the cube of the ratio of $r_{\mathrm{v}}$ to $r_{\mathrm{e}}, \rho_{\mathrm{w}}$ is the density of water and $Q$ is the scattering efficiency. $Q$ has been shown to have a constant value very close to 2 for droplet radii that are much larger than the wavelength of light concerned (B07). $c$ is the rate of increase of liquid water content $\left(q_{\mathrm{L}}\right)$ with height $\left(\mathrm{d} q_{\mathrm{L}} / \mathrm{d} z\right.$, with units $\left.\mathrm{kgm}^{-4}\right)$ and is referred to as the "condensation rate" in B07, or the "water content lapse rate" in Painemal and Zuidema (2011, hereafter PZ11). Albrecht et al. (1990) and Ahmad et al. (2013) give two alternative derivations of this quantity. $c$ depends more strongly on the temperature $(T)$ than on the pressure $(P)$. For example, the percentage change due to a pressure decrease from 850 to $650 \mathrm{hPa}$ are $15.5,12.0$ and $8.1 \%$ at temperatures of 283 , 273 and $263 \mathrm{~K}$, respectively. Thus the pressure dependence is greater at warmer temperatures. The change as the temperature decreases from 283 to $263 \mathrm{~K}$ is 47.8 and $43.2 \%$ at 850 and $650 \mathrm{hPa}$, respectively. Since $N_{\mathrm{d}}$ calculations are generally applied to low clouds only, the range of pressure of the studied clouds is likely to be smaller than that of temperature, although pressure dependence may be important for the warmest clouds. Hence, we use a constant $P$ value of $850 \mathrm{hPa}$ due to likely inaccuracies when determining $P$ from MODIS.

Although $c$ and $T$ should strictly be taken to vary with height, in this paper we use the MODIS CTT to calculate a constant $c$ value for each data point for use in Eq. (A1). Since for stratocumulus clouds the change in $T$ throughout their depth is fairly small and given the relatively weak dependence of $c$ on $T$, this makes a negligible difference. For example, using a height dependent $c$, numerical calculations show that an adiabatic cloud with $\tau=80, r_{\mathrm{e}}=21 \mu \mathrm{m}$, a cloud base pressure of $900 \mathrm{hPa}$ and a cloud base temperature of $283 \mathrm{~K}$, would be $976 \mathrm{~m}$ thick with $N_{\mathrm{d}}=60.2 \mathrm{~cm}^{-3}$ after making the assumption that $N_{\mathrm{d}}$ is constant with height. Approximating $c$ as a constant, calculated from the cloud top temperature and a pressure of $850 \mathrm{hPa}$ (the constant value assumed in the calculations in this paper), results in an underprediction of $N_{\mathrm{d}}$ of only $2 \%$. Since this example represents a very thick stratocumulus cloud, the error in most circumstances is likely to be smaller than this.

This derivation of $N_{\mathrm{d}}$ requires a number of assumptions to be made about the sampled clouds. The first assumption is that $N_{\mathrm{d}}$ is constant with height throughout the cloud depth. However, there is good observational evidence that this is the case for a number of different types of clouds in a variety of different regions, but in particular for warm stratiform clouds (PZ11; Miles et al., 2000; Wood, 2005a).

Another assumption is that the clouds are adiabatic, or some constant fraction of adiabatic. For all but the deepest of clouds this equates to $q_{\mathrm{L}}$ increasing linearly with height. There have been in situ and surface remote-sensing observational studies that indicate that this assumption is accurate (Albrecht et al., 1990; Zuidema et al., 2005). From aircraft observations made in the SE Pacific region PZ11 found linear $q_{\mathrm{L}}$ profiles within stratocumulus that on average had $c$ values that were $70 \%$ of adiabatic, i.e.

$c_{\text {observed }}=f c_{\text {adiabatic }}$,

with $f=0.7$ being the subadiabaticity. This is approximately consistent with Wood (2005b), which shows $f$ values of 0.6-0.9 for single-layer stratocumulus. The results there suggest that $f=0.7$ is a more appropriate choice than the $f=1.0$ value that would apply to a fully adiabatic cloud. It is possible that this subadiabaticity fraction varies depending upon region; cloud type and depth; and upon conditions, e.g. whether the cloud is precipitating, whether ice is present, the degree of entrainment, etc. However, as can be seen from Eq. (A1) the dependence of $N_{\mathrm{d}}$ on $c$ is fairly weak, being proportional only to $c^{0.5}$.

A further assumption for which there is also good evidence is that $k$ assumes a fairly constant value. Martin et al. (1994) found a $k$ range of $0.7-0.8$. PZ11 found profile averaged $k$ values of around 0.8 , but an increase to 0.88 near cloud top. However, here we adopt the value of $k=0.8$, which was used in B07 and as the "baseline" case in PZ11.

In line with other studies, PZ11 found that, on average, MODIS $r_{\mathrm{e}}$ values were 15-20\% too large compared to the in situ observations. Potential reasons for this discrepancy are discussed in Sect. 5. However, PZ11 showed that when the $f$ and $k$ values mentioned above ( $=0.7$ and 0.88 , respectively), which represent a modification of the more conventional values (1.0 and 0.8), were applied in Eq. (A1), along with a constant correction factor that reduced $r_{\mathrm{e}}$ by $15 \%$, the resulting $N_{\mathrm{d}}$ values were only $6 \%$ smaller than those obtained using the standard MODIS $r_{\mathrm{e}}$ and the more conventional values of the $f$ and $k$ parameters. This was because the $f$ and $k$ modifications mostly cancelled out the $r_{\mathrm{e}}$ modifications. In the present study we leave these factors unchanged from the conventional values, but note that the $N_{\mathrm{d}}$ values will be similar to those that would be produced if the adjusted parameters that were suggested in PZ11 were applied. The same would not be true for other derived quantities such as LWP and cloud thickness (see formulae in B07). 
Appendix B: Discussion on the effect of the diurnal cycle on our results

Observations show that subtropical stratocumulus clouds tend to thicken at night-time due to the absence of short-wave heating at cloud top (Wood, 2012) and that this is accompanied by increased drizzle rates. Such clouds generally reach their thickest in the early morning, just before the sun comes up. Thus, for those clouds we might expect $\tau$ to be highest at this time due to enhanced LWP. $N_{\mathrm{d}}$ effects might also influence $\tau$, although for adiabatic clouds (see Appendix A) $\tau \propto N_{\mathrm{d}}^{1 / 3} \mathrm{LWP}^{5 / 6}$ and thus more sensitivity to LWP might be expected. However, $r_{\mathrm{e}}$ is more sensitive to $N_{\mathrm{d}}$ changes than LWP changes since $r_{\mathrm{e}} \propto N_{\mathrm{d}}^{-1 / 3} \mathrm{LWP}^{1 / 6}$. There are no measurements of the diurnal cycle of $N_{\mathrm{d}}$ in stratocumulus known to the authors. The $N_{\mathrm{d}}$ diurnal cycle is likely to be complicated due to competing (but relatively weak) sources and sinks of $N_{\mathrm{d}}$ at night-time; enhanced updrafts and surface fluxes may lead to an additional $N_{\mathrm{d}}$ source, whereas enhanced precipitation is likely to cause $N_{\mathrm{d}}$ depletion. However, we note that the timescales that govern boundary layer sources and sinks of CCN are of the order of a few days (Wood, 2006; Wood et al., 2012) such that any change in these processes due to $\theta_{0}$ variation is likely to have a damped effect upon CCN concentrations and thus likely upon $N_{\mathrm{d}}$. The additional LWP at night-time in stratocumulus would likely lead to an increase in $r_{\mathrm{e}}$ in the absence of $N_{\mathrm{d}}$ changes.
However, the clouds in our study region may behave differently than those in other stratocumulus regions. In summer, at the high latitudes of our study area, the difference in $\theta_{0}$ between midday and $12 \mathrm{~h}$ later is much less than at lower latitudes and this is likely to reduce the amplitude of the diurnal cycles of cloud properties such as LWP, $\tau, r_{\mathrm{e}}$ and $N_{\mathrm{d}}$. Measurements of the diurnal cycles of $\tau, r_{\mathrm{e}}$ and $N_{\mathrm{d}}$ are lacking for the clouds in the region of our study. However, O'Dell et al. (2008) reported that LWP diurnal amplitudes in the area were $<\sim 10-20 \%$ in July (June results were not shown), which is amongst the lowest value found globally. Other stratocumulus regions show amplitudes of 30-50\% (see also Wood et al., 2002). The local time of maximum LWP was around 03:00-06:00 LT, which is a little earlier than for other stratocumulus regions where 06:00-09:00 LT was more typical. These times are consistent with the time at which the local $\theta_{0}$ decreases to below around $70-80^{\circ}$, suggesting that at this $\theta_{0}$ short-wave heating effects start to reduce LWP due to solar heating as the Sun rises. A 10-20\% increase in LWP corresponds to an approximate increase in $\tau$ of $8-17 \%$ and an $r_{\mathrm{e}}$ increase of $\sim 1.5-3 \%$, if it is assumed that $N_{\mathrm{d}}$ stays constant. This issue, with reference to our results, is discussed in Sect. 4.3. 


\section{Appendix C: Discussion on the likelihood of ice formation in the sampled cloud population}

For clouds with temperatures throughout that are warmer than $-5^{\circ} \mathrm{C}$, de Boer et al. (2011) showed that liquid-only clouds accounted for approximately 22, 65 and $90 \%$ of clouds observed at three Arctic locations and very little iceonly cloud was observed. Whilst those locations were not near those of our study, and were not open ocean regions they likely provide some insight into the issue. As the temperature increased, the dominance of liquid-only cloud became more complete. The preponderance of mixed phase and liquid-only cloud at temperatures $>-5^{\circ} \mathrm{C}$ is also supported by the observed (at various locations worldwide) very low concentrations of ice nuclei (IN) with which to initiate primary ice formation (DeMott et al., 2010) at such relatively warm temperatures.
However, the Hallett-Mossop secondary ice multiplication mechanism is known to operate between the temperatures of -3 and $-8^{\circ} \mathrm{C}$ (Hallett and Mossop, 1974), with maximum multiplication occurring in the middle of this temperature range. Seeding of such relatively warm clouds by falling ice from clouds above, followed by Hallett-Mossop ice production is thought to be a cause of significant ice production even in Antarctic stratus clouds (Grosvenor et al., 2012) where aerosol concentrations are very low. Nevertheless, Grosvenor et al. (2012) also showed that the HallettMossop process does not always operate in all supercooled cloud, even if it is within the right temperature range. A further complication is that some evidence suggests that it is the temperature at the surface of the riming ice particle that governs the process rather than the ambient air temperature (Heymsfield and Mossop, 1984). The ice surface temperature can be warmer than the air temperature due to latent heat release from the freezing liquid. This would reduce the likelihood of the Hallett-Mossop process occurring at the temperatures considered here. 


\section{Appendix D: Sensitivity analysis of $N_{\mathrm{d}}$ changes with $\theta_{0}$ using Latin hypercube sampling}

Here we describe in detail the method used to explore the relative importance of $\tau$ and $r_{\mathrm{e}}$ changes in causing the increase in $N_{\mathrm{d}}$ between low and high $\theta_{0}$. Latin hypercube sampling (LHS) sampling was used, which allows us to include the effects of the data spread and distribution shapes on the sensitivity analysis. Using LHS we constructed pairs of $\tau$ and $r_{\mathrm{e}}$ values, each containing 100000 data points, which retained the same distribution shapes as the $\tau$ and $r_{\mathrm{e}}$ PDFs shown in Figs. 6 and 8. This is done for both the low and high $\theta_{0}$ ranges and for combinations of the two. It is also possible to introduce correlation between $\tau$ and $r_{\mathrm{e}}$ using the method of Iman and Conover (1982).

Using the constructed sample sets we calculated $N_{\mathrm{d}}$ values using Eq. (A1). The accuracy of the constructed samples was likely to have been good because the mean $N_{\mathrm{d}}$ calculated from the LHS sets was close to that calculated using the actual $N_{\mathrm{d}}$ value from the real data, although with a slight tendency to overestimate. This gives confidence in the use of these samples in the sensitivity analysis. This overestimate was reduced when the observed correlations (see Table 2) were included in the LHS sampling.

The low $\theta_{0}$ LHS set for $\tau$ was then combined with the high $\theta_{0}$ set for $r_{\mathrm{e}}$ in order to calculate a mean $N_{\mathrm{d}}$, denoted as $N_{\Delta \mathrm{re}}$. $\Delta N_{\Delta \mathrm{re}}$, which is listed in Table 1 is thus the relative change in $N_{\mathrm{d}}$ between low and high $\theta_{0}$ due to changes in $r_{\mathrm{e}}$ only:

$\Delta N_{\Delta \mathrm{re}}=100\left(N_{\Delta \mathrm{re}}-N_{\text {low }}\right) / N_{\text {low }}$,

where $N_{\text {low }}$ is the $N_{\mathrm{d}}$ value at low $\theta_{0}$. In a similar way we calculated $\Delta N_{\Delta \tau}$ using the $r_{\mathrm{e}}$ set for low $\theta_{0}$ and the $\tau$ set for high $\theta_{0}$. These values are discussed in Sect. 4.5.

As mentioned above, incorporating the correlation between $\tau$ and $r_{\mathrm{e}}$ brought the LHS $N_{\mathrm{d}}$ values closer to the actual values for the low and high $\theta_{0}$ sets. However, it is difficult to choose values for the correlation between the $\tau$ LHS set at low $\theta_{0}$ and the $r_{\mathrm{e}}$ set at high $\theta_{0}$ (and vice versa) since the correlations between $\tau$ and $r_{\mathrm{e}}$ were seen to vary with $\theta_{0}$ (Table 2). Thus, we use zero correlation here, but note that the error introduced is likely to be less than $10 \%$.
As an aside, it is interesting to note that $N_{\mathrm{d}}$ values calculated using the mean $\tau$ and $r_{\mathrm{e}}$ values of the distributions (using Eq. A1) produce lower values than the actual $N_{\mathrm{d}}$ values (using the individual $\tau$ and $r_{\mathrm{e}}$ values from the sample distributions) by between 7 and $15 \%$. This is probably due to the high degree of non-linearity in the $N_{\mathrm{d}}$ equation so that combinations of low $r_{\mathrm{e}}$ and high $\tau$ from values in the tails of the distributions lead to very large $N_{\mathrm{d}}$ values. The highest values occur for the $3.7 \mu \mathrm{m}$ band. Thus, care must be taken when using the mean $\tau$ and $r_{\mathrm{e}}$ of a set of values to calculate the mean $N_{\mathrm{d}}$ of that set. This could also have implications for the method used here whereby we use the mean $\tau$ and $r_{\mathrm{e}}$ values over a $1^{\circ} \times 1^{\circ}$ area. However, it seems likely that if $\tau$ and $r_{\mathrm{e}}$ values from very small regions (e.g. single MODIS pixels) are used then the calculated $N_{\mathrm{d}}$ might also become prone to biases due to uncertainties, heterogeneities, etc, which may become "smoothed out" by averaging over larger regions. Thus, it seems likely that there is an optimal averaging scale for $\tau$ and $r_{\mathrm{e}}$ for the calculation of $N_{\mathrm{d}}$.

Whilst using mean values may not give the correct absolute values of $N_{\mathrm{d}}$, our analysis indicates that using them for the sensitivity analysis in order to calculate $\Delta N_{\Delta \tau}$ and $\Delta N_{\Delta \text { re }}$ gives similar results to those made using the LHS distributions. This is likely because $N_{\mathrm{d}}$ calculated using the mean was wrong by the same factor at low and high $\theta_{0}$ making it likely that the sensitivity test values $N_{\Delta \tau}$ and $N_{\Delta \text { re }}$ would also be wrong by the same factor. In that case the associated relative increases in $N_{\mathrm{d}}$ from low $\theta_{0}$ values (as in Eq. D1) will be the same as for the LHS sensitivity analysis (with the assumption that the LHS method is completely accurate).

This result suggests that LHS analysis is perhaps not required for $N_{\mathrm{d}}$ sensitivity calculations since using mean values produces generally similar results. However, if the spread or shapes of the $\tau$ and $r_{\mathrm{e}}$ distributions between low and high $\theta_{0}$ were very different then this may not be the case. Additionally, significant variations in the correlation between $\tau$ and $r_{\mathrm{e}}$ distributions at low and high $\theta_{0}$ would likely lead to decreased accuracy in the sensitivity analysis for both the LHS method and that using the mean values. 
Acknowledgements. The authors would like to thank Zhibo Zhang and Dan Miller for the LUTs used in this paper, as well as Marc Michelsen for extensive technical support. We acknowledge the World Climate Research Programme's Working Group on Coupled Modelling, which is responsible for CMIP, as well as IPSL for making the CALIPSO data used in this study available. For CMIP the US Department of Energy's Program for Climate Model Diagnosis and Intercomparison provides coordinating support and led development of software infrastructure in partnership with the Global Organization for Earth System Science Portals. MODIS data were obtained from the NASA Goddard Land Processes data archive. The sea-ice data were provided by the NSDIC. This work was supported under NSF award number 0968609.

Edited by: B. Mayer

\section{References}

Ahmad, I., Mielonen, T., Portin, H. J., Arola, A., Grosvenor, D. P., Mikkonen, S., Leskinen, A., Komppula, M., Lehtinen, K. E. J., Laaksonen, A., and Romakkaniemi, S.: Long term measurements of cloud droplet concentrations and aerosol-cloud interactions in boreal boundary layer clouds, Tellus B, 65, 20138, doi:10.3402/tellusb.v65i0.20138, 2013.

Albrecht, B., Fairall, C., Thomson, D., White, A., Snider, J., and Schubert, W.: Surface-based remote-sensing of the observed and the adiabatic liquid water-content of stratocumulus clouds, Geophys. Res. Lett., 17, 89-92, doi:10.1029/G1017i001p00089, 1990.

Bennartz, R.: Global assessment of marine boundary layer cloud droplet number concentration from satellite, J. Geophys. Res.Atmos, 112, D02201, doi:10.1029/2006JD007547, 2007.

Boers, R., Acarreta, J. R., and Gras, J. L.: Satellite monitoring of the first indirect aerosol effect: retrieval of the droplet concentration of water clouds, J. Geophys. Res.-Atmos., 111, D22208, doi:10.1029/2005jd006838, 2006.

Cahalan, R., Ridgway, W., Wiscombe, W., Bell, T., and Snider, J.: The albedo of fractal stratocumulus clouds, J. Atmos. Sci., 51, 2434-2455, doi:10.1175/15200469(1994)051<2434:TAOFSC>2.0.Co;2, 1994.

Chang, F.-L. and Li, Z.: Estimating the vertical variation of cloud droplet effective radius using multispectral near-infrared satellite measurements, J. Geophys. Res.-Atmos., 107, AAC 7-1-AAC 712, doi:10.1029/2001JD000766, 2002.

Chang, F.-L. and Li, Z.: Retrieving vertical profiles of watercloud droplet effective radius: algorithm modification and preliminary application, J. Geophys. Res., 108, 4763, doi:10.1029/2003JD003906, 2003.

Chen, R., Chang, F.-L., Li, Z., Ferraro, R., and Weng, F.: Impact of the vertical variation of cloud droplet size on the estimation of cloud liquid water path and rain detection, J. Atmos. Sci., 64, 3843-3853, doi:10.1175/2007JAS2126.1, 2007.

Chepfer, H., Bony, S., Winker, D., Cesana, G., Dufresne, J. L., Minnis, P., Stubenrauch, C. J., and Zeng, S.: The GCM-oriented CALIPSO cloud product (CALIPSO-GOCCP), J. Geophys. Res.-Atmos., 115, D00H16, doi:10.1029/2009JD012251, 2010.

Davies, R.: Effect of finite geometry on 3-dimensional transfer of solar irradiance in clouds, J. Atmos. Sci., 35, 1712-1725,
doi:10.1175/1520-0469(1978)035<1712:TEOFGO>2.0.CO;2, 1978.

de Boer, G., Morrison, H., Shupe, M. D., and Hildner, R.: Evidence of liquid dependent ice nucleation in high-latitude stratiform clouds from surface remote sensors, Geophys. Res. Lett., 38, L01803, doi:10.1029/2010GL046016, 2011.

DeMott, P. J., Prenni, A. J., Liu, X., Kreidenweis, S. M., Petters, M. D., Twohy, C. H., Richardson, M. S., Eidhammer, T., and Rogers, D. C.: Predicting global atmospheric ice nuclei distributions and their impacts on climate, P. Natl. Acad. Sci. USA, 107, 11217-11222, doi:10.1073/pnas.0910818107, 2010.

Foot, J.: Some observations of the optical-properties of clouds.1. Stratocumulus, Q. J. Roy. Meteor. Soc., 114, 129-144, doi:10.1256/Smsqj.47906, 1988.

Grosvenor, D. P., Choularton, T. W., Lachlan-Cope, T., Gallagher, M. W., Crosier, J., Bower, K. N., Ladkin, R. S., and Dorsey, J. R.: In-situ aircraft observations of ice concentrations within clouds over the Antarctic Peninsula and Larsen Ice Shelf, Atmos. Chem. Phys., 12, 11275-11294, doi:10.5194/acp12-11275-2012, 2012.

Hallett, J. and Mossop, S.: Production of secondary ice particles during the riming process, Nature, 249, 26-28, doi:10.1038/249026a0, 1974.

Heymsfield, A. J. and Mossop, S. C.: Temperature dependence of secondary ice crystal production during soft hail growth by riming, Q. J. Roy. Meteor. Soc., 110, 765-770, doi:10.1002/qj.49711046512, 1984.

Hoose, C., Kristjánsson, J. E., Iversen, T., Kirkevåg, A., Seland, Ø., and Gettelman, A.: Constraining cloud droplet number concentration in GCMs suppresses the aerosol indirect effect, Geophys Res. Lett., 36, L12807, doi:10.1029/2009GL038568, 2009.

Iman, R. and Conover, W.: A distribution-free approach to inducing rank correlation among input variables, Commun. Stat. B-Simul., 11, 311-334, doi:10.1080/03610918208812265, 1982.

IPCC: Climate Change 2007 - The Physical Science Basis: Working Group I Contribution to the Fourth Assessment Report of the IPCC, Cambridge University Press, Cambridge, UK, New York, NY, USA, 2007.

King, M. D., Tsay, S.-C., Platnick, S. E., Wang, M., and Liou, K. N.: Cloud retriveal algorithms for MODIS, Optical thickness, effective particle radius, and thermodynamic phase, NASA, MODIS Algorithm Theoretical Basis document No. ATBD-MOD-05, 1997.

King, M. D., Platnick, S., Yang, P., Arnold, G., Gray, M., Riedi, J., Ackerman, S., and Liou, K.: Remote sensing of liquid water and ice cloud optical thickness and effective radius in the Arctic: application of airborne multispectral MAS data, J. Atmos. Ocean. Tech., 21, 857-875, doi:10.1175/15200426(2004)021<0857:RSOLWA>2.0.CO;2, 2004.

King, N. J. and Vaughan, G.: Using passive remote sensing to retrieve the vertical variation of cloud droplet size in marine stratocumulus: an assessment of information content and the potential for improved retrievals from hyperspectral measurements, J. Geophys. Res.-Atmos., 117, D15206, doi:10.1029/2012JD017896, 2012.

Kobayashi, T.: Effects due to cloud geometry on biases in the albedo derived from radiance measurements, J. Climate, 6, 120-128, doi:10.1175/1520-0442(1993)006<0120:EDTCGO>2.0.CO;2, 1993. 
Liang, L. and Girolamo, L. D.: A global analysis on the view-angle dependence of plane-parallel oceanic liquid water cloud optical thickness using data synergy from MISR and MODIS, J. Geophys. Res.-Atmos., 118, 2389-2403, doi:10.1029/2012JD018201, 2013.

Loeb, N. and Coakley, J.: Inference of marine stratus cloud optical depths from satellite measurements: does 1-D theory apply?, J. Climate, 11, 215-233, doi:10.1175/15200442(1998)011<0215:IOMSCO>2.0.CO;2, 1998.

Loeb, N. and Davies, R.: Observational evidence of plane parallel model biases: apparent dependence of cloud optical depth on solar zenith angle, J. Geophys. Res.-Atmos., 101, 1621-1634, doi:10.1029/95JD03298, 1996.

Loeb, N. and Davies, R.: Angular dependence of observed reflectances: a comparison with plane parallel theory, J. Geophys. Res.-Atmos., 102, 6865-6881, doi:10.1029/96JD03586, 1997.

Loeb, N., Varnai, T., and Davies, R.: Effect of cloud inhomogeneities on the solar zenith angle dependence of nadir reflectance, J. Geophys. Res.-Atmos., 102, 9387-9395, doi:10.1029/96JD03719, 1997.

Loeb, N., Varnai, T., and Winker, D.: Influence of subpixel-scale cloud-top structure on reflectances from overcast stratiform cloud layers, J. Atmos. Sci., 55, 2960-2973, doi:10.1175/15200469(1998)055<2960:IOSSCT>2.0.CO;2, 1998.

Maddux, B. C., Ackerman, S. A., and Platnick, S.: Viewing geometry dependencies in MODIS cloud products, J. Atmos. Ocean. Tech., 27, 1519-1528, doi:10.1175/2010JTECHA1432.1, 2010.

Marshak, A., Davis, A., Wiscombe, W., and Cahalan, R.: Radiative effects of sub-mean free path liquid water variability observed in stratiform clouds, J. Geophys. Res.-Atmos., 103, 19557-19567, doi:10.1029/98JD01728, 1998.

Marshak, A., Platnick, S., Varnai, T., Wen, G., and Cahalan, R.: Impact of three-dimensional radiative effects on satellite retrievals of cloud droplet sizes, J. Geophys. Res.-Atmos., 111, D09207, doi:10.1029/2005JD006686, 2006.

Martin, G., JOHNSON, D., and SPICE, A.: The measurement and parameterization of effective radius of droplets in warm stratocumulus clouds, J. Atmos. Sci., 51, 1823-1842, doi:10.1175/15200469(1994)051<1823:TMAPOE>2.0.CO;2, 1994.

Miles, N., Verlinde, J., and Clothiaux, E.: Cloud droplet size distributions in low-level stratiform clouds, J. Atmos. Sci., 57, 295-311, doi:10.1175/15200469(2000)057<0295:CDSDIL>2.0.CO;2, 2000.

Morrison, H., de Boer, G., Feingold, G., Harrington, J., Shupe, M. D., and Sulia, K.: Resilience of persistent Arctic mixed-phase clouds, Nat. Geosci., 5, 11-17, doi:10.1038/NGEO1332, 2012.

Mossop, S.: Secondary ice particle-production during rime growth - the effect of drop size distribution and rimer velocity, Q. J. Roy. Meteor. Soc., 111, 1113-1124, doi:10.1002/qj.49711147012, 1985.

Nakajima, T. and King, M.: Determination of the opticalthickness and effective particle radius of clouds from reflected solar-radiation measurements.1. Theory, J. Atmos. Sci., 47, 1878-1893, doi:10.1175/15200469(1990)047<1878:DOTOTA>2.0.CO;2, 1990.

Nakajima, T., Higurashi, A., Kawamoto, K., and Penner, J.: A possible correlation between satellite-derived cloud and aerosol mi- crophysical parameters, Geophys. Res. Lett., 28, 1171-1174, doi:10.1029/2000GL012186, 2001.

Nakajima, T. Y., Suzuki, K., and Stephens, G. L.: Droplet growth in warm water clouds observed by the A-Train - Part 1: Sensitivity analysis of the MODIS-derived cloud droplet sizes, J. Atmos. Sci., 67, 1884-1896, doi:10.1175/2009JAS3280.1, 2010a.

Nakajima, T. Y., Suzuki, K., and Stephens, G. L.: Droplet growth in warm water clouds observed by the A-Train Part 2: A multisensor view, J. Atmos. Sci., 67, 1897-1907, doi:10.1175/2010JAS3276.1, 2010b.

O’Dell, C. W., Wentz, F. J., and Bennartz, R.: Cloud liquid water path from satellite-based passive microwave observations: a new climatology over the global oceans, J. Climate, 21, 1721-1739, doi:10.1175/2007JCLI1958.1, 2008.

Oreopoulos, L.: The impact of subsampling on MODIS Level-3 statistics of cloud optical thickness and effective radius, IEEE T. Geosci. Remote, 43, 366-373, doi:10.1109/TGRS.2004.841247, 2005.

Painemal, D. and Zuidema, P.: Assessment of MODIS cloud effective radius and optical thickness retrievals over the Southeast Pacific with VOCALS-REx in situ measurements, J. Geophys. Res.-Atmos., 116, D24206, doi:10.1029/2011JD016155, 2011.

Platnick, S.: Vertical photon transport in cloud remote sensing problems, J. Geophys. Res.-Atmos., 105, 22919-22935, doi:10.1029/2000JD900333, 2000.

Platnick, S., King, M., Ackerman, S., Menzel, W., Baum, B., Riedi, J., and Frey, R.: The MODIS cloud products: algorithms and examples from Terra, IEEE T. Geosci. Remote, 41, 459-473, doi:10.1109/TGRS.2002.808301, 2003.

Quaas, J., Boucher, O., Bellouin, N., and Kinne, S.: Satellite-based estimate of the direct and indirect aerosol climate forcing, J. Geophys. Res.-Atmos., 113, D05204, doi:10.1029/2007JD008962, 2008.

Quaas, J., Ming, Y., Menon, S., Takemura, T., Wang, M., Penner, J. E., Gettelman, A., Lohmann, U., Bellouin, N., Boucher, O., Sayer, A. M., Thomas, G. E., McComiskey, A., Feingold, G., Hoose, C., Kristjánsson, J. E., Liu, X., Balkanski, Y., Donner, L. J., Ginoux, P. A., Stier, P., Grandey, B., Feichter, J., Sednev, I., Bauer, S. E., Koch, D., Grainger, R. G., Kirkevåg, A., Iversen, T., Seland, Ø., Easter, R., Ghan, S. J., Rasch, P. J., Morrison, H., Lamarque, J.-F., Iacono, M. J., Kinne, S., and Schulz, M.: Aerosol indirect effects - general circulation model intercomparison and evaluation with satellite data, Atmos. Chem. Phys., 9, 8697-8717, doi:10.5194/acp-98697-2009, 2009.

Saunders, C. and Hosseini, A.: A laboratory study of the effect of velocity on Hallett-Mossop ice crystal multiplication, Atmos. Res., 59, 3-14, doi:10.1016/S0169-8095(01)00106-5, 13th International Conference on Clouds and Precipitation, Desert Res Inst, Reno, Nevada, 14-17 August, 2000, 2001.

Seethala, C. and Horvath, A.: Global assessment of AMSR$\mathrm{E}$ and MODIS cloud liquid water path retrievals in warm oceanic clouds, J. Geophys. Res.-Atmos., 115, D13202, doi:10.1029/2009JD012662, 2010.

Shupe, M. D.: Clouds at Arctic atmospheric observatories - Part 2: Thermodynamic phase characteristics, J. Appl. Meteorol. Clim., 50, 645-661, doi:10.1175/2010JAMC2468.1, 2011. 
Tjernstrom, M.: Is there a diurnal cycle in the summer cloudcapped arctic boundary layer?, J. Atmos. Sci., 64, 3970-3986, doi:10.1175/2007JAS2257.1, 2007.

Varnai, T. and Davies, R.: Effects of cloud heterogeneities on shortwave radiation: comparison of cloud-top variability and internal heterogeneity, J. Atmos. Sci., 56, 4206-4224, doi:10.1175/15200469(1999)056<4206:EOCHOS>2.0.CO;2, 1999.

Wood, R.: Drizzle in stratiform boundary layer clouds - Part 1: Vertical and horizontal structure, J. Atmos. Sci., 62, 3011-3033, doi:10.1175/JAS3529.1, 2005a.

Wood, R.: Drizzle in stratiform boundary layer clouds - Part 1: Vertical and horizontal structure, J. Atmos. Sci., 62, 3011-3033, doi:10.1175/JAS3529.1, 2005 b.

Wood, R.: Rate of loss of cloud droplets by coalescence in warm clouds, J. Geophys. Res.-Atmos., 111, D21205, doi:10.1029/2006JD007553, 2006.

Wood, R.: Stratocumulus clouds, Mon. Weather Rev., 140, 2373 2423, doi:10.1175/MWR-D-11-00121.1, 2012.

Wood, R. and Hartmann, D.: Spatial variability of liquid water path in marine low cloud: the importance of mesoscale cellular convection, J. Climate, 19, 1748-1764, doi:10.1175/JCLI3702.1, 2006.

Wood, R., Bretherton, C. S., and Hartmann, D. L.: Diurnal cycle of liquid water path over the subtropical and tropical oceans, Geophys. Res. Lett., 29, 7-1-7-4, doi:10.1029/2002GL015371, 2002.
Wood, R., Leon, D., Lebsock, M., Snider, J., and Clarke, A. D.: Precipitation driving of droplet concentration variability in marine low clouds, J. Geophys. Res.-Atmos., 117, D19210, doi:10.1029/2012JD018305, 2012.

Zhang, Z.: On the sensitivity of cloud effective radius retrieval based on spectral method to bi-modal droplet size distribution: a semi-analytical model, J. Quant. Spectrosc. Ra., 129, 79-88, doi:10.1016/j.jqsrt.2013.05.033, 2013.

Zhang, Z. and Plantnick, S.: An assessment of differences between cloud effective particle radius retrievals for marine water clouds from three MODIS spectral bands, J. Geophys. Res.-Atmos., 116, D20215, doi:10.1029/2011JD016216, 2011.

Zhang, Z., Ackerman, A. S., Feingold, G., Platnick, S., Pincus, R., and Xue, H.: Effects of cloud horizontal inhomogeneity and drizzle on remote sensing of cloud droplet effective radius: case studies based on large-eddy simulations, J. Geophys. Res.-Atmos. 117, D19208, doi:10.1029/2012JD017655, 2012.

Zinner, T., Wind, G., Platnick, S., and Ackerman, A. S.: Testing remote sensing on artificial observations: impact of drizzle and 3-D cloud structure on effective radius retrievals, Atmos. Chem. Phys., 10, 9535-9549, doi:10.5194/acp-10-9535-2010, 2010.

Zuidema, P., Westwater, E., Fairall, C., and Hazen, D.: Ship-based liquid water path estimates in marine stratocumulus, J. Geophys. Res.-Atmos., 110, D20206, doi:10.1029/2005JD005833, 2005. 